\title{
PROJECTIVE GEOMETRY IN CHARACTERISTIC ONE AND THE EPICYCLIC CATEGORY
}

\author{
ALAIN CONNES AND CATERINA CONSANI
}

\begin{abstract}
We show that the cyclic and epicyclic categories which play a key role in the encoding of cyclic homology and the lambda operations, are obtained from projective geometry in characteristic one over the infinite semifield of maxplus integers $\mathbb{Z}_{\max }$. Finite-dimensional vector spaces are replaced by modules defined by restriction of scalars from the one-dimensional free module, using the Frobenius endomorphisms of $\mathbb{Z}_{\max }$. The associated projective spaces are finite and provide a mathematically consistent interpretation of Tits's original idea of a geometry over the absolute point. The self-duality of the cyclic category and the cyclic descent number of permutations both acquire a geometric meaning.
\end{abstract}

\section{$\S 1$. Introduction}

In this paper we establish a bridge between the combinatorial structure underlying cyclic homology and the $\lambda$-operations on one side and the framework of geometry in characteristic one on the other. The combinatorial system supporting cyclic homology and the $\lambda$-operations is best encoded by the cyclic category (see [3]) and its natural extension to the epicyclic category (see [15], [2]), which play an important role in algebraic topology and algebraic $K$-theory (see [10]). In [8], we showed the relevance of cyclic homology of schemes and the $\lambda$-operations for the cohomological interpretation of the archimedean local factors of L-functions of arithmetic varieties, therefore opening the road to applications of cyclic homology in arithmetic.

Mathematics in characteristic one has two algebraic incarnations: one is provided by the theory of semirings and semifields ${ }^{\dagger}$ supporting tropical geometry and idempotent analysis, while the other one is centered on the

\footnotetext{
Received September 17, 2013. Revised March 16, 2014. Accepted March 19, 2014.

2010 Mathematics Subject Classification. Primary 19D55; Secondary 12K10, 51E26, $20 \mathrm{~N} 20$.

$\dagger$ The notion of semifield used here is from [12] and is distinct from the notion introduced by Dickson in the study of non-Desarguesian planes.
} 
more flexible notions of hyperrings and hyperfields on which certain numbertheoretic constructions repose. In our recent work [4]-[9] we explained the relevance of these two algebraic theories to promote the development of an absolute geometry.

In this paper we provide the geometric meaning of the cyclic and the epicyclic categories in terms of a projective geometry in characteristic one, and we supply the relation of the above categories with the absolute point. In Section 3 we show that the epicyclic category $\tilde{\Lambda}$ is isomorphic to a category $\mathcal{P}_{\mathbb{F}}$ of projective spaces over the simplest infinite semistructure of characteristic one, namely, the semifield $\mathbb{F}=\mathbb{Z}_{\max }:=(\mathbb{Z} \cup\{-\infty\}, \max ,+)$ of max-plus integers (which we will denote multiplicatively). The objects of $\mathcal{P}_{\mathbb{F}}$ are projective spaces $\mathbb{P}(E)$ where the semimodules $E$ over $\mathbb{F}$ are obtained by restriction of scalars from the one-dimensional free semimodule using the injective endomorphisms of $\mathbb{F}$. These endomorphisms form the multiplicative semigroup $\mathbb{N}^{\times}$: for each integer $n \in \mathbb{N}^{\times}$the corresponding endomorphism is the Frobenius $\operatorname{Fr}_{n}: \operatorname{Fr}_{n}(x):=x^{n} \forall x \in \mathbb{F}$. We denote by $\mathbb{F}^{(n)}$ the semimodule over $\mathbb{F}$ obtained from $\mathbb{F}$ by restriction of scalars using $\operatorname{Fr}_{n} \in \operatorname{End}(\mathbb{F})$; then for $n \geq 0$ the projective spaces $\mathbb{P}\left(\mathbb{F}^{(n+1)}\right)$ provide the complete collection of objects of $\mathcal{P}_{\mathbb{F}}$. The morphisms in $\mathcal{P}_{\mathbb{F}}$ are projective classes of semilinear maps $f$ of semimodules over $\mathbb{F}$ which fulfill the condition $f^{-1}(\{0\})=\{0\}$. One also derives the definition of a full (but not faithful) functor $\mathbb{P}: \mathcal{P}_{\mathbb{F}} \longrightarrow \mathfrak{F i n}$ to the category of finite sets which associates to a semimodule $E$ over $\mathbb{F}$ the quotient space $\mathbb{P}(E)=(E \backslash\{0\}) / \mathbb{F}^{\times}$(see Remark 3.17(a)). If one restricts the construction of the morphisms in $\mathcal{P}_{\mathbb{F}}$ to maps which are linear rather than semilinear, one obtains a subcategory $\mathcal{P}_{\mathbb{F}}^{1} \subset \mathcal{P}_{\mathbb{F}}$ canonically isomorphic to the cyclic category $\Lambda$ : the inclusion functor $\mathcal{P}_{\mathbb{F}}^{1} \hookrightarrow \mathcal{P}_{\mathbb{F}}$ corresponds to the inclusion of the categories $\Lambda \subset \tilde{\Lambda}$.

It is traditional to view the category of finite sets as the limit for $q=1$ of the category of finite-dimensional vector spaces over a finite field $\mathbb{F}_{q}$, and the symmetric group $S_{n}$ as the limit case of the general linear group $\mathrm{GL}_{n}\left(\mathbb{F}_{q}\right)$. There is, however, one feature of the category of finite-dimensional vector spaces over a field which is not preserved by this analogy, namely, the selfduality provided by transposition of linear maps. Indeed, the cardinality of the set of maps $\operatorname{Hom}_{\mathfrak{F} \mathfrak{i n}}(X, Y)$ between two finite sets is a highly asymmetric function of the sets, whereas for vector spaces over $\mathbb{F}_{q}$ the cardinality of $\operatorname{Hom}_{\mathbb{F}_{q}}\left(E_{1}, E_{2}\right)$ is the symmetric function $q^{n_{1} n_{2}}$, for $n_{j}=\operatorname{dim} E_{j}(j=1,2)$.

The geometric interpretation provided in this paper of the epi/cyclic categories and of the functor $\mathbb{P}$ refines and clarifies the above correspondence. 
In Section 4 we prove that the well-known self-duality of the cyclic category is described by transposition of linear maps. On the other hand, the failure of the extension of the property of self-duality to the epicyclic category is explained by the fact that the transpose of a semilinear map fails to be semilinear when the associated morphism of fields is not surjective. In our construction, the semilinearity of the maps is encoded by the functor $\operatorname{Mod}: \mathcal{P}_{\mathbb{F}} \longrightarrow \mathbb{N}^{\times}$to the multiplicative monoid of natural numbers (viewed as a small category with a single object) which associates to a morphism $f$ in $\mathcal{P}_{\mathbb{F}}$ the integer $n \in \mathbb{N}^{\times}$such that $f(\lambda x)=\operatorname{Fr}_{n}(\lambda) x \forall \lambda \in \mathbb{F}$. The functor Mod also provides, using the functor $\mathbb{P}: \mathcal{P}_{\mathbb{F}} \longrightarrow \mathfrak{F i n}$, a geometric interpretation of the cyclic descent number of arbitrary permutations as the measure of their semilinearity (see Proposition 4.10).

One can finally formulate a mathematically consistent interpretation of Tits's original idea in [19, Section 13] of a geometry over the absolute point which is provided in our construction by the data given by the category $\mathcal{P}_{\mathbb{F}}$ $\left(\mathbb{F}=\mathbb{Z}_{\max }\right)$ and the functor $\mathbb{P}$. Note that the cardinality of the set underlying the projective space $\mathbb{P}\left(\mathbb{F}^{(n+1)}\right)$ is $n+1$ and that this integer coincides with the limit, for $q \rightarrow 1$, of the cardinality of the projective space $\mathbb{P}^{n}\left(\mathbb{F}_{q}\right)=\mathbb{P}\left(\mathbb{F}_{q}^{n+1}\right)$. The fullness of the functor $\mathbb{P}$ shows in particular that any permutation $\sigma \in S_{n+1}$ arises from a geometric morphism of projective spaces over $\mathbb{F}$.

Even though the above development of a (projective) geometry in characteristic one is formulated in terms of algebraic semistructures, we show, in Section 5, how one can obtain its counterpart in the framework of hyperstructures by applying a natural functor $-\otimes_{\mathbb{B}} \mathbf{S}$, where $\mathbf{S}$ is the smallest finite hyperfield of signs (see [6]) that minimally contains the smallest finite idempotent semifield $\mathbb{B}$. In [9] we have shown that by implementing the theory of hyperrings and hyperfields, one can parallel successfully Fontaine's $p$-adic arithmetic theory of "perfection" and subsequent Witt extension by combining a process of dequantization (to characteristic one) and a consecutive Witt construction (to characteristic zero). In view of the fact that this dequantization process needs the framework of hyperstructures to be meaningful, it seems evident that the arithmetical standpoint in characteristic one requires a very flexible algebraic theory which encompasses semistructures. On the other hand, several successful developments of the theory of semirings in linear algebra and analysis show that the context of semistructures is already adequate for many applications. The only reasonable conclusion one can draw is that for the general development of mathematics in char- 
acteristic one, we ought to keep both constructions available and select the most appropriate one in relation to the specific context in which each problem is formulated.

\section{$\S 2 . \quad$ The epicyclic category}

In this section we show that the notion of archimedean set and related category $\mathfrak{A} \mathfrak{r c}$ (that we introduced in [7]) provides a natural framework for the definition of the variants $\Lambda_{a}$ (see [2], [10]) of the cyclic category $\Lambda$ of [3] and of the epicyclic category $\tilde{\Lambda}$ (originally due to Goodwillie). These categories are defined as full subcategories of categories $\mathfrak{A r c}, \mathfrak{A r c}_{a}$, and $\mathfrak{A r c} \triangleleft \mathbb{N}^{\times}$ which will be introduced in this section and whose objects are archimedean sets. The full subcategories are obtained by restricting to objects whose underlying ordered set is the set $\mathbb{Z}$ of integers with the usual total order.

\subsection{The category $\mathfrak{A} \mathfrak{r}$ of archimedean sets}

We recall from [7, Definition 5.9] the following notion.

Definition 2.1. An archimedean set is a pair $(X, \theta)$ of a nonempty, totally ordered set $X$ and an order automorphism $\theta \in$ Aut $X$ such that $\theta(x)>x, \forall x \in X$, and fulfilling the following archimedean property:

$$
\forall x, y \in X, \exists n \in \mathbb{N}: \quad y \leq \theta^{n}(x) .
$$

For each positive integer $a \in \mathbb{N}$, we introduce the following category $\mathfrak{A r c}_{a}$.

Definition 2.2. The objects of the category $\mathfrak{A r c}_{a}$ are archimedean sets $(X, \theta)$; the morphisms $f:(X, \theta) \rightarrow\left(X^{\prime}, \theta^{\prime}\right)$ in $\mathfrak{A r c}_{a}$ are equivalence classes of maps

$$
\begin{aligned}
f: X \rightarrow X^{\prime}, \quad f(x) & \geq f(y) \quad \forall x \geq y ; \\
f(\theta(x)) & =\theta^{\prime}(f(x)), \quad \forall x \in X,
\end{aligned}
$$

where the equivalence relation identifies two such maps $f$ and $g$ if there exists an integer $m \in \mathbb{Z}$ such that $g(x)=\theta^{\prime m a}(f(x)), \forall x \in X$.

For $a=1$, we drop the index 1: $\mathfrak{A} \mathfrak{n c} \mathfrak{c}_{1}=\mathfrak{A} \mathfrak{r c}$ coincides with the category of archimedean sets.

Definition 2.3. For $n \geq 1$, we denote by $\hat{n}=(\mathbb{Z}, \theta)$ the archimedean set where $\mathbb{Z}$ is endowed with the usual order, and the order automorphism $\theta: \mathbb{Z} \rightarrow \mathbb{Z}$ is given by the translation $\theta(x)=x+n$. 
The archimedean set $\hat{n}$ gives rise to the object $[n-1]$ of $\Lambda_{a}$; the shifted indexing fits with the standard notation for the object $[n]:=\{0, \ldots, n\}$ of the simplicial category $\Delta$.

Proposition 2.4. Let $a \in \mathbb{N}$. The full subcategory of $\mathfrak{A r c}_{a}$, whose objects are the archimedean sets $\hat{n}=(\mathbb{Z}, \theta)$ for $n \geq 1$, is canonically isomorphic to the a-cyclic category $\Lambda_{a}$ considered in [2] and [10].

Proof. We fix $a$, and we let $\mathcal{C}$ be the full subcategory of $\mathfrak{A r c}_{a}$ whose objects are the archimedean sets $\hat{n}$ for $n \geq 1$. There is an inclusion functor $j: \Delta \hookrightarrow \mathcal{C}$ that is the map $[n] \mapsto \widehat{n+1}$ on the objects of the simplicial category $\Delta$ and on the morphisms $f \in \operatorname{Hom}_{\Delta}([n],[m])$ defined as follows:

$$
j(f)(x+k(n+1))=f(x)+k(m+1), \quad \forall x \in[n], k \in \mathbb{Z} .
$$

One easily checks that $j(f)^{-1}([0, m])=[0, n]$, since by construction one has $j(f)([0, n]+k(n+1)) \subset[0, m]+k(m+1), \forall k \in \mathbb{Z}$. Moreover, any morphism $\phi \in \operatorname{Hom}_{\mathfrak{A r c}_{a}}(\widehat{n+1}, \widehat{m+1})$ such that $\phi^{-1}([0, m])=[0, n]$ is of the form $j(f)$ for a unique $f \in \operatorname{Hom}_{\Delta}([n],[m])$. We introduce the cyclic permutation $\tau$ :

$$
\tau: \mathbb{Z} \rightarrow \mathbb{Z}, \quad \tau(x)=x-1, \quad \forall x \in \mathbb{Z}
$$

Note that, for each $n \geq 0, \tau$ yields an element $\tau_{n-1} \in C_{n a}=\operatorname{Aut}_{\mathfrak{A r c}_{a}}(\hat{n})$ (the index $n$ of $\tau$ is shifted by 1 ). The relation $\tau_{n-1}^{n a}=$ id follows from the definition of the equivalence relation in Definition 2.2. Let $\phi \in \operatorname{Hom}_{\mathfrak{A r c}_{a}}(\widehat{n+1}$, $\widehat{m+1})$ and $I=[0, m] \subset \mathbb{Z}$. Then $\phi^{-1}(I)$ is a finite interval $J$ because $\phi$ is increasing and $\phi(x) \rightarrow \pm \infty$ when $x \rightarrow \pm \infty$. Moreover, since for $k \in \mathbb{Z}$ (varying) the translates $I+k(m+1)$ form a partition of $\mathbb{Z}$, the same statement holds for their inverse images which are, in view of the periodicity property of the maps in $\mathfrak{A r c}_{a}$, of the form $J+k(n+1)$. Thus $J=[u, u+n]$ for some $u \in \mathbb{Z}$. It follows that there exist a unique $f \in \operatorname{Hom}_{\Delta}([n],[m])$, an integer $v$ uniquely determined modulo $(n+1) a$, and a decomposition

$$
\phi=j(f) \circ \tau_{n}^{v}
$$

This shows that the category $\mathcal{C}$ is an extension of the small simplicial category $\Delta$ by means of a new generator $\tau_{n}$ of the cyclic group $C_{(n+1) a}$, for each $n \geq 0$. Moreover, one checks, as for the cyclic category, the following relations in terms of the face maps $\delta_{j}$ and degeneracies $\sigma_{j}$ of $\Delta$, 


$$
\begin{aligned}
& \tau_{n}^{(n+1) a}=\mathrm{id}, \\
& \tau_{n} \circ \sigma_{0}=\sigma_{n} \circ \tau_{n+1}^{2}, \quad \tau_{n} \circ \sigma_{j}=\sigma_{j-1} \circ \tau_{n+1}, \quad \forall j \in\{1, \ldots, n\} \\
& \tau_{n} \circ \delta_{0}=\delta_{n}, \quad \tau_{n} \circ \delta_{j}=\delta_{j-1} \circ \tau_{n-1}, \quad \forall j \in\{1, \ldots, n\} .
\end{aligned}
$$

The uniqueness of the decomposition (2) shows that the above relations give a presentation of $\mathcal{C}$ and thus that $\mathcal{C}$ is canonically isomorphic to the $a$-cyclic category $\Lambda_{a}$ considered in [2] and [10].

\subsection{The correspondences $\bar{\Psi}_{k}$}

Let $(X, \theta)$ be an archimedean set, and let $k>0$ be an integer. Then the pair $\left(X, \theta^{k}\right)$ is also an archimedean set that we denote as

$$
\Psi_{k}(X, \theta):=\left(X, \theta^{k}\right) .
$$

A morphism $f:(X, \theta) \rightarrow\left(X^{\prime}, \theta^{\prime}\right)$ in $\mathfrak{A} \mathfrak{r c}$ fulfills (1) and hence one has $f\left(\theta^{k}(x)\right)=\theta^{\prime k}(f(x)), \forall x \in X \quad(k>0$ fixed). Thus $f$ defines a morphism $\Psi_{k}(f) \in \operatorname{Hom}_{\mathfrak{A} \mathfrak{c} \mathfrak{c}}\left(\Psi_{k}(X, \theta), \Psi_{k}\left(X^{\prime}, \theta^{\prime}\right)\right)$. However, the two maps $f$ and $\theta^{\prime} \circ f$ (which define the same morphism in the category $\mathfrak{A} \mathfrak{r c}$ ) are in general no longer equivalent as morphisms $\Psi_{k}(X, \theta) \rightarrow \Psi_{k}\left(X^{\prime}, \theta^{\prime}\right)$. Thus, rather than a functor, one derives more precisely a correspondence $\bar{\Psi}_{k}: \mathfrak{A r c} \rightarrow \mathfrak{A} \mathfrak{r c}$ that satisfies the following properties.

Proposition 2.5.

(i) Let $h \in \operatorname{Hom}_{\mathfrak{A r c}}\left((X, \theta),\left(X^{\prime}, \theta^{\prime}\right)\right)$; then for a fixed positive integer $k>0$ the set

$$
\bar{\Psi}_{k}(h):=\left\{\Psi_{k}(f) \mid f \in h\right\}
$$

is finite with exactly $k$ elements (we write $f \in h$ when $f$ is in the equivalence class of the morphism $h$ ).

(ii) Let $g, h$ be composable morphisms in $\mathfrak{A} \mathfrak{r}$; then one has

$$
\bar{\Psi}_{k}(g \circ h)=\bar{\Psi}_{k}(g) \circ \bar{\Psi}_{k}(h):=\left\{u \circ v \mid u \in \bar{\Psi}_{k}(g), v \in \bar{\Psi}_{k}(h)\right\} .
$$

(iii) For any pair of positive integers $k, k^{\prime}: \bar{\Psi}_{k} \circ \bar{\Psi}_{k^{\prime}}=\bar{\Psi}_{k k^{\prime}}$.

Proof. (i) Let $f:(X, \theta) \rightarrow\left(X^{\prime}, \theta^{\prime}\right)$ be a morphism in $\mathfrak{A} \mathfrak{r c}$. Then the composite $\theta^{\prime k} \circ f$ is equivalent to $f$ in the set $\operatorname{Hom}_{\mathfrak{A r c}}\left(\Psi_{k}(X, \theta), \Psi_{k}\left(X^{\prime}, \theta^{\prime}\right)\right)$, while the class of $f$ in $\operatorname{Hom}_{\mathfrak{A} \mathfrak{r c}}\left((X, \theta),\left(X^{\prime}, \theta^{\prime}\right)\right)$ is represented by $\theta^{\prime m} \circ f$, for $m \in \mathbb{Z}$. Thus $\bar{\Psi}_{k}(h)$ is the finite set of classes of $\theta^{\prime m} \circ f$, for $m \in\{0, \ldots, k-1\}$. These elements are pairwise inequivalent since the maps $\theta^{\prime m} \circ f$ are pairwise distinct for $m \in \mathbb{Z}$. 
(ii) Let $h \in \operatorname{Hom}_{\mathfrak{A} \mathfrak{c} \mathfrak{c}}\left((X, \theta),\left(X^{\prime}, \theta^{\prime}\right)\right)$, and let $g \in \operatorname{Hom}_{\mathfrak{A} \mathfrak{c} c}\left(\left(X^{\prime}, \theta^{\prime}\right),\left(X^{\prime \prime}, \theta^{\prime \prime}\right)\right)$. Let $g^{\prime} \in g$ and $h^{\prime} \in h$ be maps in the corresponding equivalence classes (fulfilling (1)). Then $g^{\prime} \circ h^{\prime} \in g \circ h$, and one also has by construction $\Psi_{k}\left(g^{\prime} \circ h^{\prime}\right)=$ $\Psi_{k}\left(g^{\prime}\right) \circ \Psi_{k}\left(h^{\prime}\right)$. By replacing $h^{\prime}$ by $\theta^{\prime n} \circ h^{\prime}$ and $g^{\prime}$ by $\theta^{\prime \prime m} \circ g^{\prime}$, one substitutes $z=g^{\prime} \circ h^{\prime}$ with $\theta^{\prime \prime a} \circ z$ for $a=n+m$. Note that only the class of $a$ modulo $k$ matters for the corresponding morphism: $\Psi_{k}(X, \theta) \rightarrow \Psi_{k}\left(X^{\prime \prime}, \theta^{\prime \prime}\right)$.

(iii) For any morphism $f:(X, \theta) \rightarrow\left(X^{\prime}, \theta^{\prime}\right)$ in $\mathfrak{A} \mathfrak{r c}$, one easily checks that $\Psi_{k} \circ \Psi_{k^{\prime}}(f)=\Psi_{k k^{\prime}}(f)$.

2.3. Two functors $\mathfrak{A r c}_{a} \longrightarrow \mathfrak{A r c}_{b}$ when $b \mid a$

The correspondences $\bar{\Psi}_{k}: \mathfrak{A} \mathfrak{r c} \rightarrow \mathfrak{A} \mathfrak{r} \mathfrak{c}$ are best described in terms of two functors

$$
P, \Psi_{k}: \mathfrak{A r c}_{k} \longrightarrow \mathfrak{A} \mathfrak{r c}
$$

which we now describe in slightly more general terms.

Let $a, b \in \mathbb{N}$ : when $b \mid a$, the functor $P: \mathfrak{A r c}_{a} \longrightarrow \mathfrak{A r c}_{b}$ is defined as the natural forgetful functor. Namely, $P$ is the identity on objects and associates to an equivalence class (see Definition 2.2) of morphisms $f \in \operatorname{Hom}_{\mathfrak{A r c}_{a}}((X, \theta)$, $\left.\left(X^{\prime}, \theta^{\prime}\right)\right)$ the unique class it defines in $\operatorname{Hom}_{\mathfrak{A} \mathfrak{r} \mathfrak{c}_{b}}\left((X, \theta),\left(X^{\prime}, \theta^{\prime}\right)\right)$.

The definition of $\Psi_{k}$ given in Section 2.2 determines, for every integer $t>0$, a functor

$$
\Psi_{k}: \mathfrak{A r c}_{k t} \longrightarrow \mathfrak{A} \mathfrak{r} \mathfrak{c}_{t} .
$$

In fact the two maps $f$ and $\theta^{\prime k t} \circ f$, which define the same morphism in the category $\mathfrak{A r c}_{k t}$, are equivalent as morphisms in the set $\operatorname{Hom}_{\mathfrak{A r c}_{t}}\left(\Psi_{k}(X, \theta)\right.$, $\left.\Psi_{k}\left(X^{\prime}, \theta^{\prime}\right)\right)$. One thus obtains the following commutative diagram where the lower horizontal arrow is the correspondence $\bar{\Psi}_{k}$ :

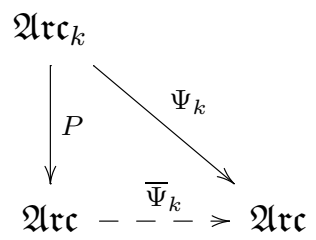

\subsection{The category $\mathfrak{A} \mathfrak{r} \triangleleft \mathbb{N}^{\times}$}

Proposition 2.5 describes an action of the multiplicative monoid $\mathbb{N}^{\times}$by correspondences on $\mathfrak{A} \mathfrak{c}$. Next we define the category $\mathfrak{A} \mathfrak{c} \triangleleft \mathbb{N}^{\times}$obtained as the semidirect product of $\mathfrak{A} \mathfrak{r}$ by this action. Its objects are the same as 
those of $\mathfrak{A} \mathfrak{r}$. At the level of morphisms, instead, we adjoin, for each object $(X, \theta)$ of $\mathfrak{A} \mathfrak{c} \mathfrak{c}$ and each positive integer $k$, a new morphism

$$
\psi_{k}: \Psi_{k}(X, \theta) \rightarrow(X, \theta)
$$

which fulfills the relations

$$
f \circ \psi_{k}=\psi_{k} \circ g, \quad \forall g \in \bar{\Psi}_{k}(f), \forall f \in \operatorname{Hom}_{\mathfrak{A} \mathfrak{c} c}\left((X, \theta),\left(X^{\prime}, \theta^{\prime}\right)\right)
$$

and

$$
\psi_{k} \circ \psi_{k^{\prime}}=\psi_{k k^{\prime}}, \quad \forall k, k^{\prime}>0 .
$$

This construction is precisely achieved as follows.

Definition 2.6. The objects of the category $\mathfrak{A} \mathfrak{r} \triangleleft \mathbb{N}^{\times}$are the archimedean

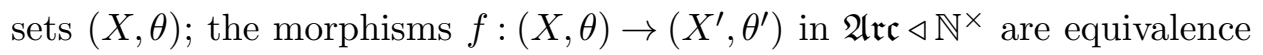
classes of maps

$$
\begin{aligned}
& f: X \rightarrow X^{\prime}, \quad f(x) \geq f(y) \quad \forall x \geq y ; \\
& \exists k \in \mathbb{N}, \quad f(\theta(x))=\theta^{\prime k}(f(x)), \quad \forall x \in X,
\end{aligned}
$$

where the equivalence relation identifies two such maps $f$ and $g$ if there exists an integer $m \in \mathbb{Z}$ such that $g(x)=\theta^{\prime m}(f(x)), \forall x \in X$.

We check that the equivalence relation is compatible with the composition of maps in $\mathfrak{A} \mathfrak{r} \triangleleft \mathbb{N}^{\times}$.

Let $f_{j}:(X, \theta) \rightarrow\left(X^{\prime}, \theta^{\prime}\right)(j=1,2)$ and $g:\left(X^{\prime}, \theta^{\prime}\right) \rightarrow\left(X^{\prime \prime}, \theta^{\prime \prime}\right)$ be two morphisms. One has $f_{1} \sim f_{2} \Leftrightarrow f_{2}(x)=\theta^{\prime m}\left(f_{1}(x)\right), \forall x \in X$ and for some $m \in \mathbb{Z}$. It follows (since $g$ fulfills (8)) that $g \circ f_{2}(x)=g\left(\theta^{\prime m}\left(f_{1}(x)\right)\right)=\theta^{\prime \prime k m} g\left(f_{1}(x)\right)$, thus $g \circ f_{2} \sim g \circ f_{1}$.

The next proposition shows that the category $\mathfrak{A} \mathfrak{c} \triangleleft \mathbb{N}^{\times}$has exactly the expected properties of a semidirect product of $\mathfrak{A} \mathfrak{r} \mathfrak{c}$ by the correspondences $\bar{\Psi}_{k}$.

Proposition 2.7.

(i) The category $\mathfrak{A} \mathfrak{r c}$ is a subcategory of $\mathfrak{A} \mathfrak{r} \triangleleft \mathbb{N}^{\times}$.

(ii) The map $\rho: \operatorname{Hom}_{\mathfrak{A} \mathfrak{c} \triangleleft \mathbb{N}^{\times}}\left((X, \theta),\left(X^{\prime}, \theta^{\prime}\right)\right) \rightarrow \mathbb{N}^{\times}, \rho(f)=k \in \mathbb{N}^{\times}$such that (8) holds, describes at the morphisms level a functor to the category $\mathbb{N}^{\times}$with a single object $* \operatorname{Hom}(*, *)=\mathbb{N}^{\times}$,

$$
\rho:\left(\mathfrak{A r c} \triangleleft \mathbb{N}^{\times}\right) \longrightarrow \mathbb{N}^{\times} .
$$


(iii) For any archimedean set $(X, \theta)$ and positive integer $k$, the identity map $\operatorname{id}_{X}$ determines a morphism $\psi_{k} \in \operatorname{Hom}_{\mathfrak{A} \mathfrak{r} \triangleleft \mathbb{N} \times}\left(\Psi_{k}(X, \theta),(X, \theta)\right)$ which fulfills the relations (6) and (7).

(iv) Every morphism $f \in \operatorname{Hom}_{\mathfrak{A} \mathfrak{c} \triangleleft \mathbb{N} \times}\left((X, \theta),\left(X^{\prime}, \theta^{\prime}\right)\right)$ is of the form

$$
f=\psi_{k} \circ h, \quad k=\rho(f), h \in \operatorname{Hom}_{\mathfrak{A} \mathfrak{r c}}\left((X, \theta), \Psi_{k}\left(X^{\prime}, \theta^{\prime}\right)\right) .
$$

Proof. (i) The categories $\mathfrak{A} \mathfrak{r c}$ and $\mathfrak{A} \mathfrak{r} \triangleleft \mathbb{N}^{\times}$share the same objects, and by construction one has

$$
\operatorname{Hom}_{\mathfrak{A} \mathfrak{r c}}\left((X, \theta),\left(X^{\prime}, \theta^{\prime}\right)\right) \subset \operatorname{Hom}_{\mathfrak{A} \mathfrak{c} \triangleleft \mathbb{N} \times}\left((X, \theta),\left(X^{\prime}, \theta^{\prime}\right)\right) .
$$

(ii) Since the action of $\theta^{\prime}$ on $X^{\prime}$ is free, the value of $k$ for which (8) holds is uniquely determined. One also checks easily that $\rho(f \circ g)=\rho(f) \rho(g)$.

(iii) The identity map $u=\operatorname{id}_{X}$ fulfills $u \circ \theta^{k}=\theta^{k} \circ u$, and thus defines a morphism $\psi_{k}$ in the set $\operatorname{Hom}_{\mathfrak{A} \mathfrak{c} \triangleleft \mathbb{N} \times}\left(\Psi_{k}(X, \theta),(X, \theta)\right)$. By applying the definition of the equivalence relation for morphisms as in Definition 2.6, one has $\psi_{k} \circ \theta^{j} \sim \psi_{k}$ for all $j \in \mathbb{Z}$. One thus obtains the equality $f \circ \psi_{k}=\psi_{k} \circ g$, $\forall g \in \bar{\Psi}_{k}(f)$. The relations (6) and (7) are easily verified.

(iv) Let $f:(X, \theta) \rightarrow\left(X^{\prime}, \theta^{\prime}\right)$ in $\mathfrak{A} \mathfrak{r c} \triangleleft \mathbb{N}^{\times}$. Then $f$ gives an element $h \in$ $\operatorname{Hom}_{\mathfrak{A} \mathfrak{r}}\left((X, \theta), \Psi_{k}\left(X^{\prime}, \theta^{\prime}\right)\right)$ whose definition depends upon the choice of $f$ in its class. Replacing $f$ by $\theta^{\prime j} \circ f$ has the effect of replacing $h$ by $\theta^{\prime j} \circ h$ whose class in $\operatorname{Hom}_{\mathfrak{A} \mathfrak{c}}\left((X, \theta), \Psi_{k}\left(X^{\prime}, \theta^{\prime}\right)\right)$ depends only on the residue of $j$ modulo $k$.

Proposition 2.8. The full subcategory of $\mathfrak{A} \mathfrak{r} \triangleleft \mathbb{N}^{\times}$whose objects are the archimedean sets $(\mathbb{Z}, \theta)$ ( $\mathbb{Z}$ endowed with the usual order) is canonically isomorphic to the epicyclic category $\tilde{\Lambda}$.

Proof. Let $\mathrm{Sd}_{\mathrm{k}}: \Delta \longrightarrow \Delta$ be the edgewise subdivision functor (see, e.g., [2]) which maps the object $[n-1]$ to $[k n-1]$ and a morphism $f \in$ $\operatorname{Hom}_{\Delta}([m-1],[n-1])$ to its $k$-fold concatenation

$$
\operatorname{Sd}_{\mathrm{k}}(f)=f \perp f \perp \cdots \perp f \in \operatorname{Hom}_{\Delta}([k m-1],[k n-1]) .
$$

We recall (see [2, Definition 1.1]) that the epicyclic category $\tilde{\Lambda}$ is defined by adjoining to the cyclic category $\Lambda$, new morphisms $\pi_{n}^{k}:[k(n+1)-1] \rightarrow[n]$ for $n \geq 0, k \geq 1$, which fulfill the following relations:

(i) $\pi_{n}^{1}=\mathrm{id}_{\mathrm{n}}, \pi_{n}^{\ell} \circ \pi_{\ell(n+1)-1}^{k}=\pi_{n}^{k \ell}$;

(ii) $\alpha \pi_{m}^{k}=\pi_{n}^{k} \operatorname{Sd}_{\mathrm{k}}(\alpha)$, for any $\alpha \in \operatorname{Hom}_{\Delta}([m],[n])$;

(iii) $\tau_{n} \pi_{n}^{k}=\pi_{n}^{k} \tau_{k(n+1)-1}$. 
In terms of the archimedean sets $\hat{n}$ which we identify with $[n-1]$ (see Definition 2.3), the map $f \in \operatorname{Hom}_{\Delta}([m-1],[n-1])$ lifts uniquely to a map $\tilde{f}: \mathbb{Z} \rightarrow \mathbb{Z}$ such that $\tilde{f}(x+m)=\tilde{f}(x)+n, \forall x \in \mathbb{Z}$. Notice that $\tilde{f}$ agrees with $f$ on $\{0,1, \ldots, m-1\}$. Moreover, $\tilde{f}$ is a morphism of archimedean sets, and the class of $\Psi_{k}(\tilde{f})$ is the same as the class of the $k$-fold concatenation $\widetilde{\operatorname{Sd}}_{\mathrm{k}}(f)$. This shows that one obtains the required isomorphism of categories by extending the isomorphism of Proposition 2.4 (for $a=1$ ) to the full subcategory of $\mathfrak{A} \mathfrak{r c} \triangleleft \mathbb{N}^{\times}$whose objects are the $\hat{n}$, by mapping the morphism $\psi_{k} \in \operatorname{Hom}_{\mathfrak{A} \mathfrak{c} \triangleleft \mathbb{N} \times}\left(\Psi_{k}(\hat{n}), \hat{n}\right)$ to $\pi_{n-1}^{k}$.

REMARK 2.9. The epicyclic category $\tilde{\Lambda}$ used in this paper is originally due to T. Goodwillie and described in [2], but it does not correspond to the notion of epicyclic space applied in [14].

\subsection{The functor $\mathfrak{F}:\left(\mathfrak{A r c} \triangleleft \mathbb{N}^{\times}\right) \longrightarrow$ Sets}

In the following we denote by $\mu_{a}$ the multiplicative group of $a$ th roots of unity in $\mathbb{C}$. By $\mathfrak{S e t s}_{a}$ we denote the category of sets endowed with a free action of $\mu_{a}$, and with morphisms given by $\mu_{a}$-equivariant maps. For $(X, \theta) \in \operatorname{Obj}(\mathfrak{A} \mathfrak{r c})$, we consider the orbit space of the action of $\theta^{a}$ on $X$

$$
\mathfrak{F}_{a}(X, \theta):=X / \theta^{a \mathbb{Z}},
$$

endowed with the free action of $\mu_{a}$ generated by the action of $\theta$ on $\mathfrak{F}_{a}(X, \theta)$.

Proposition 2.10.

(i) For $a=1$, one has a functor $\mathfrak{F}=\mathfrak{F}_{1}:\left(\mathfrak{A} \mathfrak{r} \triangleleft \triangleleft \mathbb{N}^{\times}\right) \longrightarrow$ Sets.

(ii) For any integer $a>1$, one has a functor $\mathfrak{F}_{a}: \mathfrak{A r c}_{a} \longrightarrow \mathfrak{S e t}_{a}$.

Proof. (i) Let $f:(X, \theta) \rightarrow\left(X^{\prime}, \theta^{\prime}\right)$ be a morphism in $\mathfrak{A} \mathfrak{r c} \triangleleft \mathbb{N}^{\times}$(thus fulfilling (8)). Then given two points $x, y=\theta^{m}(x)$ on the same orbit of the action of $\theta$ on $X$, the points $f(x)$ and $f(y)=\theta^{\prime k m}(f(x))$ are on the same orbit of the action of $\theta^{\prime}$ on $X^{\prime}$. This shows that $\mathfrak{F}_{1}$ transforms a morphism $f \in \operatorname{Hom}_{\mathfrak{A} \mathfrak{c} \triangleleft \mathbb{N} \times}\left((X, \theta),\left(X^{\prime}, \theta^{\prime}\right)\right)$ into a map of sets; this association is also compatible with the composition of morphisms.

(ii) By definition of the equivalence relation as in Definition 2.2 for $f \in \operatorname{Hom}_{\mathfrak{A r c}_{a}}\left((X, \theta),\left(X^{\prime}, \theta^{\prime}\right)\right)$, the induced map of sets $X / \theta^{a \mathbb{Z}} \rightarrow X^{\prime} / \theta^{\prime a \mathbb{Z}}$ is independent of the choice of $f$ in its equivalence class. Moreover, the equivariance condition (1) ensures that the induced map of sets is $\mu_{a^{-}}$ equivariant. 
We will now follow the effect of the functors $P$ and $\Psi_{k}$ as in Section 2.3 in terms of the categories $\mathfrak{S e t s}_{a}$. When $b \mid a(a, b \in \mathbb{N})$, there is a canonical inclusion $\mu_{b} \subset \mu_{a}$. With $a=k b(k \in \mathbb{N})$, the subgroup $\mu_{b}$ is the range of the group endomorphism $\mu_{a} \rightarrow \mu_{a}, u \mapsto u^{k}$. This determines a natural restriction functor

$$
\text { Res : } \mathfrak{S e t s}_{a} \longrightarrow \mathfrak{S e t s}_{b}
$$

which does not alter the underlying set and restricts the action of the roots of unity $\mu_{a}$ to the subgroup $\mu_{b}$. This restriction functor corresponds to the functor $\Psi_{k}: \mathfrak{A r c}_{a} \longrightarrow \mathfrak{A r c}_{b}$ of (4), that is, the following diagram commutes:

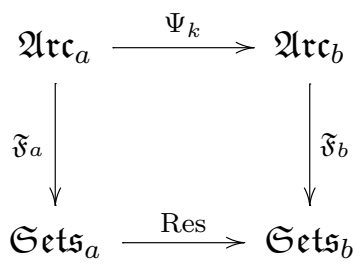

To check the commutativity of the above diagram, we note that the set underlying $\mathfrak{F}_{b}\left(\Psi_{k}(X, \theta)\right)$ is the orbit space of the action of $\theta^{k b}$ on $X$ and that this coincides with the set underlying $\mathfrak{F}_{a}(X, \theta)$.

When $a=k b$, one also has an extension of scalars functor

$$
-\times_{\mu_{a}} \mu_{b}: \mathfrak{S e t s}_{a} \longrightarrow \mathfrak{S e t s}_{b}
$$

which associates to an object $Y$ of $\mathfrak{S e t s}_{a}$ its quotient $Y \times{ }_{\mu_{a}} \mu_{b}$ for the action of the subgroup $\mu_{k} \subset \mu_{a}$. This functor corresponds to the functor $P: \mathfrak{A} \mathfrak{r c} \mathfrak{c}_{a} \longrightarrow \mathfrak{A} \mathfrak{r c} \mathfrak{c}_{b}$, that is, the following diagram commutes:

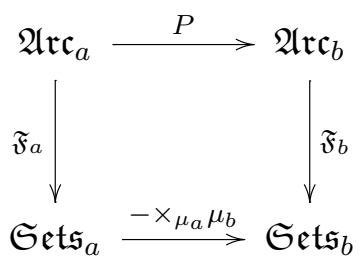

REMARK 2.11. It is customary to interpret the category $\mathfrak{S e t}_{a}$ as the category of vector spaces over $\mathbb{F}_{1^{a}}$, where $\mathbb{F}_{1^{a}}$ plays the role of the limit for $q \rightarrow 1$ of the finite fields $\mathbb{F}_{q^{a}}$. However, this analogy has its limitations since, for instance, the classical duality between vector spaces over fields does 
not apply here because, for vector spaces $V$ and $W$ over $\mathbb{F}_{1^{a}}$ of respective dimensions $n$ and $m$, the cardinality of the space of morphisms is

$$
\#\left(\operatorname{Hom}_{\mathbb{F}_{1} a}(V, W)\right)=(a m)^{n},
$$

which is not a symmetric function of $n$ and $m$. In Section 4 we will explain how the classical duality is restored for the cyclic category $\Lambda$ using the framework of projective geometry in characteristic one which we now describe.

\section{§3. $\tilde{\Lambda}$ and projective geometry over the semifield $\mathbb{Z}_{\max }$}

In ordinary projective geometry the maps between projective spaces $\mathbb{P}\left(E_{j}\right)=\left(E_{j} \backslash\{0\}\right) / K_{j}^{\times}$over fields $K_{j}(j=1,2)$ are induced by semilinear maps of vector spaces $E_{j}$ (see [11]). Recall that a map $f: E_{1} \rightarrow E_{2}$ between two vector spaces is called semilinear if it is additive and if there exists a homomorphism of fields $\sigma: K_{1} \rightarrow K_{2}$ such that $f(\lambda x)=\sigma(\lambda) f(x)$ $\forall \lambda \in K_{1}$ and $\forall x \in E_{1}$. This notion extends verbatim to the context of semifields, where by a semifield we mean a commutative semiring $K$ in which the nonzero elements form a group under multiplication (see [12, Example 4.25, p. 52]). Note that in a semiring the existence of an additive inverse is no longer required (see $[12, \mathrm{I}]$ ). Note, moreover, that homomorphisms of fields are automatically injective, and we will require injectivity of $\sigma: K_{1} \rightarrow K_{2}$ in the context of semifields.

By a semimodule $E$ over a semifield $K$ we mean (see [12, Chapter 14], [13, Chapter 5]) a commutative monoid $(E,+)$ with additive identity $0 \in E$, endowed with an action of $K$ such that $\forall \lambda, \mu \in K$ and $\forall x, y \in E$, one has

$$
\begin{gathered}
\lambda(x+y)=\lambda x+\lambda y, \quad(\lambda+\mu) x=\lambda x+\mu x, \\
(\lambda \mu) x=\lambda(\mu x), \quad 0 x=0, \quad 1 x=x .
\end{gathered}
$$

A map $f: E_{1} \rightarrow E_{2}$ between two semimodules over semifields $K_{j}$ is called semilinear if it is additive and if there exists an injective homomorphism of semifields $\sigma: K_{1} \rightarrow K_{2}$ such that $f(\lambda x)=\sigma(\lambda) f(x) \forall \lambda \in K_{1}$ and $\forall x \in E_{1}$. Two such maps $f, f^{\prime}$ are projectively equivalent when there exists $\lambda \in K_{2}$, $\lambda \neq 0$, such that $f^{\prime}(x)=\lambda f(x), \forall x \in E_{1}$.

Definition 3.1. Let $\mathfrak{P r o j}$ be the category whose objects are pairs $(K, E)$ made by a semifield $K$ and a semimodule $E$ over $K$ and whose morphisms $\left(K_{1}, E_{1}\right) \rightarrow\left(K_{2}, E_{2}\right)$ are pairs $(\sigma, h)$, where $\sigma: K_{1} \rightarrow K_{2}$ is an injective semifield homomorphism and $h$ is a projective class of additive semilinear 
maps $f: E_{1} \rightarrow E_{2}$ such that $f^{-1}(\{0\})=\{0\}$ and $f(\lambda x)=\sigma(\lambda) f(x) \forall \lambda \in K_{1}$, $\forall x \in E_{1}$.

Proposition 3.2. The assignment which maps a pair $(K, E) \in \operatorname{Obj}(\mathfrak{P r o j})$ to the corresponding projective space (as set) $\mathbb{P}(E):=(E \backslash\{0\}) / K^{\times}$and a morphism in $\mathfrak{P r o j}$ to the induced map of sets defines a covariant functor $\mathbb{P}: \mathfrak{P r o j} \longrightarrow$ Sets.

Proof. Let $f: E_{1} \rightarrow E_{2}$ be a semilinear map such that $f^{-1}(\{0\})=\{0\}$. For $x \in E_{1} \backslash\{0\}$, the class of $f(x) \in\left(E_{2} \backslash\{0\}\right) / K_{2}^{\times}$does not change if one replaces $x$ by $\lambda x$ for $\lambda \in K_{1}^{\times}$or if one replaces $f$ by $\mu f$ for $\mu \in K_{2}^{\times}$.

The assignment which associates to an object $(K, E)$ of $\mathfrak{P r o j}$ the semifield $K$, and to a morphism $(\sigma, h)$ the map $\sigma$ (as in Definition 3.1), defines a functor Mod from $\mathfrak{P r o j}$ to the category of semifields.

Definition 3.3. Let $K$ be a semifield. We denote by $\mathbb{P}_{K}$ the full subcategory of $\mathfrak{P r o j}$ whose objects are semimodules over $K$. We denote by $\mathbb{P}_{K}^{1}$ the subcategory of $\mathbb{P}_{K}$ with the same objects as $\mathbb{P}_{K}$ and whose morphisms are pairs $(\sigma, f)$, where $\sigma$ is the identity on $K$ (i.e., morphisms in $\mathbb{P}_{K}^{1}$ are given by projective classes of linear maps).

Thus, by definition, the objects of $\mathbb{P}_{K}$ are the objects $X=(K, E)$ of $\mathfrak{P r o j}$ such that $\operatorname{Mod}(X)=K$ and the morphisms $\alpha$ of $\mathbb{P}_{K}^{1}$ are such that $\operatorname{Mod}(\alpha)=\operatorname{id}_{K}$.

Next, we recall the most used definition of rank of a semimodule (see, e.g., [12, Chapter 14, p. 153]), and we also introduce the notion of free rank that generalizes, in the context of semistructures, the classical notion of the largest cardinality of a free system.

Definition 3.4. Let $E$ be a finitely generated semimodule over a semifield $K$.

(a) The $\operatorname{rank} \operatorname{rk}(E)$ is the smallest positive integer $n$ such that there exists a set of generators of $E$ of cardinality $n$.

(b) The free $\operatorname{rank} \underline{\mathrm{rk}}(E)$ is the largest positive integer $n$ such that there exists a free subsemimodule of $E$ of rank $n$.

Following [1, Exercise 16, A II.181], one can show that if $\operatorname{rk}(E)$ and $\underline{\operatorname{rk}}(E)$ are both finite, then $\underline{\operatorname{rk}}(E) \leq \operatorname{rk}(E)$. 


\subsection{The semifield $\mathbb{B}$ and the simplicial category $\Delta$}

Unlike the classical case of vector spaces over fields, a finitely generated semimodule $E$ over a semifield $K$ is not necessarily isomorphic to $K^{n}$, for some $n$. This change of behavior arises already in the simplest example of the idempotent semifield $K=\mathbb{B}=(\{0,1\},+, \cdot)$. Here the term "idempotent" means that $x+x=x, \forall x \in K$, or equivalently that $1+1=1$, which we view as reflecting the fact that one works in characteristic one. It is known (see $[12$, Chapter 4, Example 4.28]) that $\mathbb{B}$ is the only finite idempotent semifield.

Let $E$ be a semimodule over $\mathbb{B}$. Since $1+1=1$ in $\mathbb{B}$, it follows from (9) that $x+x=x, \forall x \in E$ so that, as a monoid, $E$ is idempotent, and we will use the notation $x+y=x \vee y$ for the sum of two elements of $E$. The canonical preorder of the commutative monoid $E$, defined by (see $[13,3.3$, p. 12])

$$
x \leq y \Longleftrightarrow \exists z \in E, \quad y=x \vee z,
$$

is then an order relation (see [13, Proposition 3.4.5]), and moreover one has $x \leq y \Longleftrightarrow x \vee y=y$.

The partially ordered set $(E, \leq)$ is a semilattice with a smallest element 0 , and the join of any two elements $x, y \in E$ is $x \vee y$. Conversely, given a semilattice $X$, one defines a semimodule $X^{\vee}$ over $\mathbb{B}$ by adjoining to $X$ a smallest element as follows.

Definition 3.5. Let $X$ be a semilattice. One lets $X^{\vee}=X \cup\{0\}$ be the set endowed with the following binary operation $\vee$ :

$$
x \vee y:=\operatorname{join}(x, y), \quad \forall x, y \in X, \quad 0 \vee x=x \vee 0=x, \quad \forall x \in X^{\vee}
$$

It is easy to see that the last two equations of (9) uniquely define the action of $\mathbb{B}$ on $X^{\vee}$ and that this action fulfills the other equations of (9) since $x \vee x=x, \forall x \in X^{\vee}$.

Definition 3.6 (see [13, p. 5]). A commutative monoid $E$ is selective if and only if one has $x+y \in\{x, y\}, \forall x, y \in E$.

By [13, Proposition 3.4.7], a commutative monoid is selective if and only if it is idempotent and the canonical order is total.

The following statement determines a complete list of the finitely generated semimodules of free rank 1 over $\mathbb{B}$ and their categorical interpretation. 


\section{Proposition 3.7.}

(i) Let $E$ be a $\mathbb{B}$-semimodule. Then $E$ is selective if and only if $\underline{\mathrm{rk}}(E)=1$.

(ii) For each $n \in \mathbb{N}$, there exists a unique up to canonical isomorphism $\mathbb{B}$ semimodule $E=\mathbb{B}^{(n, 1)}$ such that $\operatorname{rk}(E)=n$ and $\underline{\operatorname{rk}}(E)=1$. One has $\mathbb{B}^{(n, 1)}=X^{\vee}$ for $X=\{1, \ldots, n\}$ as a totally ordered set.

(iii) The following properties hold for the semimodules $\mathbb{B}^{(n, 1)}$ :

(a) $\mathbb{B}^{(n, 1)}$ is of minimal cardinality among the $\mathbb{B}$-semimodules of rank $n$;

(b) $\mathbb{B}^{(n, 1)}$ is a projective semimodule over $\mathbb{B}$ equal to the range of a projection matrix $P \in M_{n}(\mathbb{B}), P^{2}=P$;

(c) the semiring of endomorphisms $\operatorname{End}_{\mathbb{B}}\left(\mathbb{B}^{(n, 1)}\right)$ is isomorphic to $P M_{n}(\mathbb{B}) P$.

(iv) The simplicial category $\Delta$ is canonically isomorphic to the full subcategory $\mathcal{P}_{\mathbb{B}} \subset \mathbb{P}_{\mathbb{B}}$ whose objects are the semimodules $\mathbb{B}^{(n, 1)}$ and the morphisms are (projective classes of) linear maps $f$ such that $f^{-1}(\{0\})=$ $\{0\}$.

Proof. (i) Let $E$ be a $\mathbb{B}$-semimodule such that $\underline{\operatorname{rk}}(E)=1$. Then $x \vee y \in$ $\{x, y\}$ for any two nonzero distinct elements $x, y \in E$, since otherwise one could construct a free subsemimodule of $E$ of rank 2 . It follows that $E$ is selective. Conversely, if $E$ is selective it does not contain a copy of $\mathbb{B}^{2}$ and thus $\underline{\operatorname{rk}}(E)=1$.

(ii) The rank of $E=X^{\vee}$ for $X$ a totally ordered set is $\operatorname{rk}(E)=\operatorname{card} X$. Thus if $\operatorname{rk}(E)=n$, one has $E=\mathbb{B}^{(n, 1)}=X^{\vee}$ with $X=\{1, \ldots, n\}$ as a totally ordered set.

(iii) We show that (a), (b), and (c) hold.

(a) The cardinality of $\mathbb{B}^{(n, 1)}$ is $n+1$ and is the minimal cardinality among $\mathbb{B}$-semimodules of rank $n$, since any such semimodule contains at least 0 and the $n$ generators. Let $E$ be a $\mathbb{B}$-semimodule of rank $n$, and assume that the cardinality of $E$ is $n+1$; then $x \vee y \in\{x, y\}$ for any $x, y \in E$, since otherwise one could remove $x \vee y$ from the set of generators. Thus it follows that $E$ is selective, and thus $E=\mathbb{B}^{(n, 1)}$.

(b) One has

(11) $\quad \mathbb{B}^{(n, 1)}=P\left(\mathbb{B}^{n}\right), \quad P=\left(\begin{array}{ccccc}1 & 0 & 0 & \ldots & 0 \\ 1 & 1 & 0 & \ldots & 0 \\ 1 & 1 & 1 & \ldots & 0 \\ & \ldots & & \ldots & \ldots \\ 1 & 1 & 1 & \ldots & 1\end{array}\right) \in M_{n}(\mathbb{B}), \quad P^{2}=P$ 
since the image $P\left(\left\{e_{j}\right\}\right)$ of the canonical basis $\left\{e_{j}\right\}$ of $\mathbb{B}^{n}$ is the decreasing sequence $P\left(\left\{e_{j}\right\}\right)=\left\{\bigvee_{i \leq j} e_{i}\right\}$. This shows that $\mathbb{B}^{(n, 1)}$ is a finitely generated and projective semimodule of rank at most $n$. By (10) any subset of $X=$ $\{1, \ldots, n\}$ is stable under the binary operation $\vee$, and this shows that the rank of $X^{\vee}$ is equal to $n$.

(c) The set of endomorphisms $\operatorname{End}_{\mathbb{B}}\left(\mathbb{B}^{n}\right)$ forms a semiring isomorphic to the semiring of matrices $M_{n}(\mathbb{B})$ (see [12]). Given $T \in \operatorname{End}_{\mathbb{B}}\left(\mathbb{B}^{(n, 1)}\right)$, the composite $T \circ P$ defines an element of $\operatorname{End}_{\mathbb{B}}\left(\mathbb{B}^{n}\right)$.

(iv) There is a natural functor which associates to a totally ordered set $X$ the semimodule $X^{\vee}$ over $\mathbb{B}$. The morphisms $f \in \operatorname{Hom}_{\mathbb{B}}\left(X^{\vee}, Y^{\vee}\right)$ such that $f^{-1}(\{0\})=\{0\}$ are the nondecreasing maps from $X$ to $Y$. By applying this construction to the skeleton of the category of totally ordered finite sets, we obtain a functor from the simplicial category $\Delta$ to the category $\mathcal{P}_{\mathbb{B}}$. This functor is fully faithful and hence it defines an isomorphism of categories $\Delta \cong \mathcal{P}_{\mathbb{B}}$.

Remark 3.8. (a) Claim (iv) of Proposition 3.7 does not change if one replaces linear maps by projective classes of linear maps since $\mathbb{B}^{\times}=\{1\}$. In fact, since $\mathbb{B}$ has no nontrivial endomorphism, linearity and semilinearity are equivalent notions in this context.

(b) The subsemimodules of rank $k$ of $X^{\vee}$ are determined by the subsets of $X$ of cardinality $k$. Thus the cardinality of the set of subsemimodules of rank $k$ of $\mathbb{B}^{(n, 1)}$ is given by the binomial coefficient $\left(\begin{array}{l}n \\ k\end{array}\right)$. This is in agreement with the limit, as $q \rightarrow 1$, of the cardinality of the Grassmannian of vector subspaces of dimension $k$ in an $n$-dimensional vector space over the finite field $\mathbb{F}_{q}$.

(c) The free semimodule $\mathbb{B}^{n}$ has cardinality $2^{n}$ which grows exponentially with $n$, while the cardinality of $\mathbb{B}^{(n, 1)}$ is $n+1$ which is linear in $n$.

\subsection{Semimodules over $\mathbb{Z}_{\max }$ and archimedean sets}

Let $\mathbb{F}=\mathbb{Z}_{\text {max }}:=(\mathbb{Z} \cup\{-\infty\}, \max ,+)$ be the semifield of tropical integers: we will denote it multiplicatively, thus the elements of $\mathbb{F}$ are either 0 or a power $u^{n}$ for $n \in \mathbb{Z}$, where $u$ here is a symbol. The idempotent addition $\vee$ is such that $u^{n} \vee u^{m}=u^{k}$, with $k=\sup (n, m)$. The multiplication is the usual one: $u^{n} u^{m}=u^{n+m}$. The semifield $\mathbb{F}$ is isomorphic to the subsemifield of $\mathbb{R}_{+}^{\max }$ generated by an arbitrary element greater than 1 of $\mathbb{R}_{+}^{\max }$. Here $\mathbb{R}_{+}^{\max }$ is the multiplicative version for $\mathbb{R}_{\max }:=(\mathbb{R} \cup\{-\infty\}$, $\max ,+)$.

In this section we interpret the category $\mathfrak{A} \mathfrak{r}$ and the functor $\mathfrak{F}=\mathfrak{F}_{1}$ : $\mathfrak{A} \mathfrak{r} \longrightarrow \mathfrak{S e t s}$ in terms of the category $\mathbb{P}_{\mathbb{F}}^{1}$ of semimodules over $\mathbb{F}=\mathbb{Z}_{\max }$. 
An archimedean set $(X, \theta)$ defines a semimodule $(X, \theta)^{\vee}$ over $\mathbb{F}$ as follows.

Proposition 3.9. Let $(X, \theta)$ be an archimedean set. Let $(X, \theta)^{\vee}=(X \cup$ $\{0\}, \theta)$ be endowed with the binary operation

$$
x \vee y:=\sup (x, y) \quad \forall x, y \in X, \quad 0 \vee x=x \vee 0=x \quad \forall x \in X \cup\{0\} .
$$

The action of $\mathbb{F}$ on $(X, \theta)^{\vee}$ given by

$$
u^{n} x:=\theta^{n}(x) \quad \forall x \in X, n \in \mathbb{Z}, \quad 0 x=0 \quad \forall x \in X \cup\{0\},
$$

endows $(X, \theta)^{\vee}$ with the structure of semimodule over $\mathbb{F}$.

Proof. The condition $\theta(x)>x, \forall x \in X$, of Definition 2.1 shows that

$$
(a \vee b) x=a x \vee b x, \quad \forall x \in X \cup\{0\}, a, b \in \mathbb{F} .
$$

Moreover, the linearity property

$$
a(x \vee y)=a x \vee a y, \quad \forall x, y \in X \cup\{0\}, a \in \mathbb{F}
$$

holds since $\theta$ is an order automorphism.

Proposition 3.10. There is a fully faithful functor $\vee: \mathfrak{A} \mathfrak{c c} \longrightarrow \mathbb{P}_{\mathbb{F}}^{1}$, $(X, \theta) \mapsto(X, \theta)^{\vee}$ mapping morphisms in $\mathfrak{A} \mathfrak{r} \mathfrak{c}$ to projective classes of linear maps $f$ such that $f^{-1}(\{0\})=\{0\}$.

Proof. We know from Proposition 3.9 that if $(X, \theta)$ is an archimedean set, then $(X, \theta)^{\vee}$ is a semimodule over $\mathbb{F}$. Next we show how to define the functor $f \mapsto f^{\vee}$ on morphisms. Let $f:(X, \theta) \rightarrow\left(X^{\prime}, \theta^{\prime}\right)$ be a morphism in $\mathfrak{A} \mathfrak{r c}$ (thus fulfilling (1)); then we extend $f$ at 0 by $f(0)=0$ and obtain an $\mathbb{F}$-linear map $f^{\vee}:(X, \theta)^{\vee} \rightarrow\left(X^{\prime}, \theta^{\prime}\right)^{\vee}$. By construction, one has $f^{-1}(\{0\})=$ $\{0\}$. Moreover, replacing $f$ by $\theta^{\prime m} \circ f$ does not alter the projective class of $f^{\vee}:(X, \theta)^{\vee} \rightarrow\left(X^{\prime}, \theta^{\prime}\right)^{\vee}$ since it replaces $f^{\vee}$ by $u^{m} f^{\vee}$. In this way one obtains a functor $\mathfrak{A} \mathfrak{r} \mathfrak{c} \longrightarrow \mathbb{P}_{\mathbb{F}}^{1}$. It is faithful by construction, so it remains to show that it is full. Let $h:(X, \theta)^{\vee} \rightarrow\left(X^{\prime}, \theta^{\prime}\right)^{\vee}$ be an $\mathbb{F}$-linear map such that $h^{-1}(\{0\})=\{0\}$. Then the restriction of $h$ to $X$ defines a map $f:(X, \theta) \rightarrow$ $\left(X^{\prime}, \theta^{\prime}\right)$ that fulfills $(1)$. This shows the required surjectivity of the functor on morphisms.

REMARK 3.11. Any nonzero morphism $h:(X, \theta)^{\vee} \rightarrow\left(X^{\prime}, \theta^{\prime}\right)^{\vee}$ fulfills $h^{-1}(\{0\})=\{0\}$. Indeed, assume that $h^{-1}(\{0\})$ contains an element $x \in X$; then we prove that $h(y)=0 \forall y \in X$. The archimedean property shows that for any $y \in X$ there exists an integer $n$ such that $y \leq \theta^{n}(x)$. It follows that $h(y) \leq \theta^{\prime n}(h(x))=0$, and thus $h(y)=0$. 


\subsection{Geometric interpretation of the functor $\mathfrak{F}_{1}$}

In the above geometric terms, the functor $\mathfrak{F}_{1}: \mathfrak{A r c} \longrightarrow$ Sets is a special case of the functor $\mathbb{P}: \mathfrak{P r o j} \longrightarrow$ Sets of Proposition 3.2; that is, the following diagram commutes:

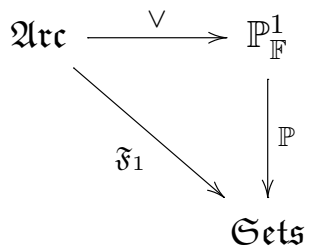

One may wonder what geometric structure remains after passing from a semimodule $E$ to the set $\mathbb{P}(E)$. In ordinary projective geometry (where $E$ would be a vector space over a field), this structure is given by the map $(x, y) \mapsto \ell(x, y)$ which associates to a pair of distinct points of a projective space the line determined by them. Then the axioms of projective geometry characterize the obtained structures in the Desarguesian case.

In the above framework of projective geometry over $\mathbb{F}=\mathbb{Z}_{\max }$, there is a similar geometric structure: the "abstract circle" described by Moerdijk. By definition an abstract circle $C$ is given by the following data:

$$
C=\left(P, S, \partial_{0}, \partial_{1}, 0,1, *, \cup\right)
$$

Here $P$ and $S$ are sets, $\partial_{j}: S \rightarrow P$ are maps as well as $P \ni x \rightarrow 0_{x} \in S$ and $P \ni x \rightarrow 1_{x} \in S, *: S \rightarrow S$ is an involution, and $\cup$ is a partially defined map from a subset of $S \times S$ to $S$. Here $P$ plays the role of the set of points of the geometry, while $S$ plays the role of the set of lines, or rather segments. In order to qualify as an abstract circle, the data (14) have to fulfill certain axioms (see [7]). It follows from these axioms that given two points $x \neq y \in P$, there exists a unique segment $s \in S$ such that $\partial_{0}(s)=x$, $\partial_{1}(s)=y$.

As shown in [7], there is a natural functor $\mathfrak{Q}$ which associates to an object $(X, \theta)$ of the category $\mathfrak{A} \mathfrak{c}$ an abstract circle $X / \theta$ and establishes in this way an equivalence of categories.

The abstract circle $X / \theta=\left(P, S, \partial_{0}, \partial_{1}, 0,1, *, \cup\right)$ associated to an archimedean set $(X, \theta)$ is obtained as follows:

- $P:=X / \sim$ is the orbit space for the action of $\mathbb{Z}$ on $X$ given by powers of $\theta$, that is, $P=\mathbb{P}(E):=(E \backslash\{0\}) / \mathbb{F}^{\times}$for $E$ semimodule over $\mathbb{F}$; 
- $S$ is the orbit space for the action of $\mathbb{Z}$ on the set of pairs $(x, y) \in X^{2}$, with $x \leq y \leq \theta(x)$;

- $\partial_{0}(x, y)=x, \partial_{1}(x, y)=y$;

- $0_{x}=(x, x), 1_{x}=(x, \theta(x))$;

- $(x, y)^{*}=(y, \theta(x))$;

- $(x, y) \cup(y, z)=(x, z)$, provided that $x \leq y \leq z \leq \theta(x)$.

It remains to be seen how to relax the conditions fulfilled by the morphisms in the category of abstract circles so that the above discussion extends to the category $\mathfrak{A} \mathfrak{r} \triangleleft \mathbb{N}^{\times}$and the diagram below commutes:

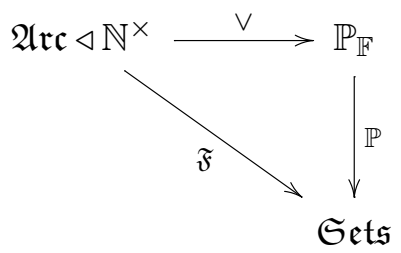

\subsection{Restriction of scalars}

In this section we implement the semigroup of endomorphisms of the semifield $\mathbb{F}=\mathbb{Z}_{\max }$ to define a functor restriction of scalars for semimodules $E$ over $\mathbb{F}$. To each integer $n \in \mathbb{N}^{\times}$corresponds an endomorphism $\operatorname{Fr}_{n} \in$ $\operatorname{End}(\mathbb{F})$ given by $\operatorname{Fr}_{n}(x):=x^{n} \forall x \in \mathbb{F}$. Moreover, one has the following.

LEMma 3.12. The map $n \mapsto \mathrm{Fr}_{n}$ is an isomorphism of semigroups: $\mathbb{N}^{\times} \rightarrow$ $\operatorname{End}(\mathbb{F}) \backslash\{\iota\}$, where $\iota \in \operatorname{End}(\mathbb{F})$ is the trivial endomorphism with range $\mathbb{B} \subset$ $\mathbb{F}$. Any injective endomorphism of $\mathbb{F}$ is equal to $\operatorname{Fr}_{n}$ for a unique $n \in \mathbb{N}^{\times}$.

Let $E$ be a semimodule over $\mathbb{F}$, and let $n \in \mathbb{N}^{\times}$. Since $\operatorname{Fr}_{n}: \mathbb{F} \rightarrow \mathbb{F}$ is a homomorphism of semifields, one can associate to $E$ the semimodule $F_{n}(E)$ over $\mathbb{F}$ with the same underlying additive structure but with a redefined multiplication by elements of $\mathbb{F}$ as follows:

$$
\lambda . \xi:=\operatorname{Fr}_{n}(\lambda) \xi
$$

Since $\mathrm{Fr}_{n}$ is not surjective, this restriction of scalars fails to pass unambiguously to projective classes of $\mathbb{F}$-linear maps $f$ with $f^{-1}(\{0\})=\{0\}$. Indeed, the ambiguity is retained by the group $\mathbb{F}^{\times} / \operatorname{Fr}_{n}\left(\mathbb{F}^{\times}\right) \cong \mu_{n}$.

3.5. Semilinear maps and $\mathfrak{A} \mathfrak{r c} \triangleleft \mathbb{N}^{\times}$

Next, we extend Proposition 3.10 to the category $\mathfrak{A} \mathfrak{r c} \triangleleft \mathbb{N}^{\times}$. 
Proposition 3.13. The functor $\vee: \mathfrak{A} \mathfrak{c} \triangleleft \mathbb{N}^{\times} \longrightarrow \mathbb{P}_{\mathbb{F}},(X, \theta) \mapsto(X, \theta)^{\vee}$, is fully faithful.

Proof. Recall (see Definition 2.6) that the objects of the category $\mathfrak{A} \mathfrak{r c} \triangleleft \mathbb{N}^{\times}$ are archimedean sets, while the morphisms are equivalence classes of maps which fulfill (8). The condition $f(\theta(x))=\theta^{\prime k}(f(x)), \forall x \in X$ implies that extending $f$ by $f(0)=0$, one obtains an $\mathbb{F}$-semilinear map $f^{\vee}:(X, \theta)^{\vee} \rightarrow$ $\left(X^{\prime}, \theta^{\prime}\right)^{\vee}$, with $f^{\vee}(\lambda \xi)=\operatorname{Fr}_{k}(\lambda) f^{\vee}(\xi) \forall \lambda \in \mathbb{F}$ and $\xi \in(X, \theta)^{\vee}$. Since any injective morphism $\sigma: \mathbb{F} \rightarrow \mathbb{F}$ is equal to $\operatorname{Fr}_{k}$ for some $k \in \mathbb{N}^{\times}$(see Lemma 3.12), the proof of Proposition 3.10 applies verbatim. It shows that the obtained functor, from the category $\mathfrak{A} \mathfrak{r} \triangleleft \mathbb{N}^{\times}$to the category of semimodules over $\mathbb{F}$ with morphisms given by projective classes of semilinear maps $f$ such that $f^{-1}(\{0\})=\{0\}$, is fully faithful.

\subsection{The epicyclic category $\tilde{\Lambda}$ and projective geometry over $\mathbb{Z}_{\max }$}

We first investigate the structure of the semimodules $E$ over $\mathbb{F}$ obtained from the one-dimensional free semimodule by restriction of scalars using the endomorphisms of $\mathbb{F}$.

\section{Proposition 3.14.}

(i) The semimodules over $\mathbb{F}$ of the form $(\mathbb{Z}, \theta)^{\vee}$ (i.e., coming from archimedean sets of the form $(\mathbb{Z}, \theta)$ ) are obtained by restriction of scalars and they are all of the form $F_{n}(\mathbb{F})=: \mathbb{F}^{(n)}$, where the integer $n \in \mathbb{N}$ is such that $\theta(x)=x+n, \forall x \in \mathbb{Z}$.

(ii) The functor $\mathbb{P}: \mathbb{P}_{\mathbb{F}} \rightarrow \mathfrak{S e t s}, E \longrightarrow \mathbb{P}(E)$, establishes a bijection between the subsemimodules of $\mathbb{F}^{(n)}$ and the subsets of $\mathbb{P}\left(\mathbb{F}^{(n)}\right)$.

(iii) Let $E$ be a subsemimodule of $\mathbb{F}^{(n)}$. Then $E$ is isomorphic to $\mathbb{F}^{(k)}$, where $k$ is the rank of $E$.

Proof. (i) The archimedean set $\hat{n}=(\mathbb{Z}, \theta)$ with $\theta(x)=x+n, \forall x \in \mathbb{Z}$, is such that $(\mathbb{Z}, \theta)^{\vee}=F_{n}(\mathbb{F})$ as follows from the definition of the restriction of scalars.

(ii) One has by construction $\mathbb{P}(E) \subset \mathbb{P}\left(\mathbb{F}^{(n)}\right)$, and this gives an injection between the set of subsemimodules of $\mathbb{F}^{(n)}$ and the set of subsets of $\mathbb{P}\left(\mathbb{F}^{(n)}\right)$. To prove that this map is surjective, it is enough to show that given a subset $Y \subset \mathbb{P}\left(\mathbb{F}^{(n)}\right)$ with $k$ elements there exists a morphism $f: \mathbb{F}^{(k)} \rightarrow \mathbb{F}^{(n)}$ such that the range of $\mathbb{P}(f)$ is $Y$. This statement will also prove (iii) provided that $f$ is an isomorphism with its range. From (i) one has $\mathbb{F}^{(n)}=(\mathbb{Z}, \theta)^{\vee}$, where $\theta(x)=x+n \forall x \in \mathbb{Z}$. The subset $\{0, \ldots, n-1\} \subset \mathbb{Z}$ is a fundamental domain 
for the action of $\theta$ and gives an identification $\mathbb{P}\left(\mathbb{F}^{(n)}\right)=\{0, \ldots, n-1\}$. One has $Y=\left\{y_{0}, \ldots, y_{k-1}\right\} \subset\{0, \ldots, n-1\}$, where $y_{0}<y_{1}<\cdots<y_{k-1}$. Let

$$
f(x):=y_{\bar{x}}+n E(x / k), \quad \forall x \in \mathbb{Z},
$$

where $\bar{x} \in\{0, \ldots, n-1\}$ is the residue of $x$ modulo $n$, and $E(z)$ is the integer part of $z \in \mathbb{R}$. Then $f: \mathbb{Z} \rightarrow \mathbb{Z}$ is an increasing map and fulfills $f(x+k)=$ $f(x)+n \forall x \in \mathbb{Z}$. Thus $f$ defines an injective morphism $f: \mathbb{F}^{(k)} \rightarrow \mathbb{F}^{(n)}$ such that the range of $\mathbb{P}(f)$ is $Y$.

The next statement shows that the epicyclic category $\tilde{\Lambda}$ encodes projective geometry over the semifield $\mathbb{F}=\mathbb{Z}_{\max }$, where the projective spaces $\mathbb{P}(E)$ are constructed from the semimodules $E=\mathbb{F}^{(n)}$ over $\mathbb{F}$.

THEOREM 3.15 .

(i) The epicyclic category $\tilde{\Lambda}$ is canonically isomorphic to the full subcategory $\mathcal{P}_{\mathbb{F}} \subset \mathbb{P}_{\mathbb{F}}$ whose objects are obtained from the one-dimensional free semimodule $\mathbb{F}=\mathbb{Z}_{\max }$ by restriction of scalars using the Frobenius endomorphisms of $\mathbb{F}$.

(ii) The cyclic category $\Lambda \subset \tilde{\Lambda}$ is faithfully embedded as the subcategory $\mathcal{P}_{\mathbb{F}}^{1} \subset$ $\mathcal{P}_{\mathbb{F}}$ with the same objects of $\mathcal{P}_{\mathbb{F}}$ and whose morphisms are induced by linear maps.

Proof. (i) The statement follows from Proposition 3.13, Proposition 2.8, and Proposition 3.14. Thus the object $[n-1]$ of $\tilde{\Lambda}$ corresponds canonically to $F_{n}(\mathbb{F})=\mathbb{F}^{(n)}$, and Proposition 3.13 determines the canonical isomorphism.

Statement (ii) follows from Proposition 3.10.

Next, we investigate how the inclusion $\Delta \subset \Lambda$ of the simplicial category into the cyclic category arises from extension of scalars from $\mathbb{B}$ to $\mathbb{F}=\mathbb{Z}_{\max }$. First, we need to relate the $\mathbb{F}$-semimodule $\mathbb{F}^{(n)}$ with the semimodule $\mathbb{B}^{(n, 1)}$ $(n \in \mathbb{N})$. Both pairs $\left(\mathbb{B}, \mathbb{B}^{(n, 1)}\right)$ and $\left(\mathbb{F}, \mathbb{F}^{(n)}\right)$ are objects of the category $\mathfrak{P r o j}$ as in Definition 3.1.

Let $\iota: \mathbb{B} \rightarrow \mathbb{F}$ be the unique homomorphism of semifields. By construction $\mathbb{F}^{(n)}=(\mathbb{Z}, \theta)^{\vee}$, where $\theta(x)=x+n \forall x \in \mathbb{Z}$.

Let $\iota_{n}: \mathbb{B}^{(n, 1)} \rightarrow \mathbb{F}^{(n)}$ be $\gamma^{\vee}$, where $\gamma$ is the unique increasing map which identifies the finite ordered set $\mathbb{B}^{(n, 1)} \backslash\{0\}$ with the subset $\{0, \ldots, n-1\} \subset \mathbb{Z}$.

Proposition 3.16.

(i) The pair $\left(\iota, \iota_{n}\right)$ defines a morphism in $\mathfrak{P r o j}$. 
(ii) Let $f \in \operatorname{Hom}_{\mathcal{P}_{\mathbb{B}}}\left(\mathbb{B}^{(n, 1)}, \mathbb{B}^{(m, 1)}\right)$. Then there exists a unique $\tilde{f} \in$ $\operatorname{Hom}_{\mathcal{P}_{\mathbb{F}}^{1}}\left(\mathbb{F}^{(n)}, \mathbb{F}^{(m)}\right)$ such that the following diagram commutes in $\mathfrak{P r o j}$ :

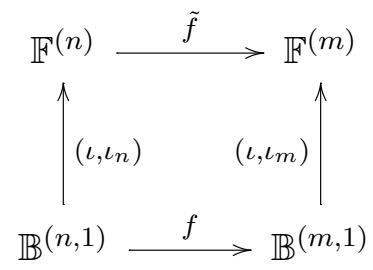

(iii) The functor $\mathcal{P}_{\mathbb{B}} \longrightarrow \mathcal{P}_{\mathbb{F}}^{1}, f \mapsto \tilde{f}$, corresponds to the canonical inclusion $\Delta \subset \Lambda$.

Proof. (i) By construction $\iota_{n}: \mathbb{B}^{(n, 1)} \rightarrow \mathbb{F}^{(n)}$ is additive and $\mathbb{B}$-linear, thus the pair $\left(\iota, \iota_{n}\right)$ defines a morphism $\left(\iota, \iota_{n}\right) \in \operatorname{Hom}_{\mathfrak{P r o j}}\left(\left(\mathbb{B}, \mathbb{B}^{(n, 1)}\right),\left(\mathbb{F}, \mathbb{F}^{(n)}\right)\right)$ which is also represented for each $k \in \mathbb{Z}$ by the projectively equivalent pair $\left(\iota, u^{k} \iota_{n}\right)$.

(ii) We identify $\mathbb{B}^{(n, 1)} \backslash\{0\}$ with the subset $\{0, \ldots, n-1\} \subset \mathbb{Z}$. The morphism $f \in \operatorname{Hom}_{\mathcal{P}_{\mathbb{B}}}\left(\mathbb{B}^{(n, 1)}, \mathbb{B}^{(m, 1)}\right)$ is given by a unique nondecreasing map (we still denote it by $f$ ) $f:\{0, \ldots, n-1\} \rightarrow\{0, \ldots, m-1\}$. To prove the existence of $\tilde{f}$, one defines the map $g: \mathbb{Z} \rightarrow \mathbb{Z}$ by

$$
g(j+k n)=f(j)+k m, \quad \forall j \in\{0, \ldots, n-1\}, k \in \mathbb{Z} .
$$

One then gets that $g^{\vee} \in \operatorname{Hom}_{\mathcal{P}_{\mathbb{F}}^{1}}\left(\mathbb{F}^{(n)}, \mathbb{F}^{(m)}\right)$ and that the diagram (15) is commutative. This proves the existence of $g=\tilde{f}$. To prove the uniqueness of $\tilde{f}$, note that every nonzero element of $\mathbb{F}^{(n)}$ is of the form $x=u^{k} \iota_{n}(y)$ for some $y \in \mathbb{B}^{(n, 1)}$. Thus if the diagram (15), with $h$ instead of $\tilde{f}$, commutes in $\mathfrak{P r o j}$, there exists $\ell \in \mathbb{Z}$ such that $h(j+k n)=f(j)+k m+\ell m \forall j \in\{0, \ldots$, $n-1\}, k \in \mathbb{Z}$, and this shows that $h$ is in the same projective class as the above $g$.

(iii) By construction $g=\tilde{f}$ fulfills (16), and this determines on the morphisms the canonical embedding $\Delta \subset \Lambda$.

REMARK 3.17. (a) When applied to the morphism $\left(\iota, \iota_{n}\right) \in \operatorname{Hom}_{\mathfrak{P r o j}}((\mathbb{B}$, $\left.\left.\mathbb{B}^{(n, 1)}\right),\left(\mathbb{F}, \mathbb{F}^{(n)}\right)\right)$, the functor $\mathbb{P}$ of Proposition 3.2 determines a bijection

$$
\mathbb{P}\left(\mathbb{B}^{(n, 1)}\right) \stackrel{\mathbb{P}\left(\iota, \iota_{n}\right)}{\rightarrow} \mathbb{P}\left(\mathbb{F}^{(n)}\right)
$$


Thus, as a set, the projective space does not change by implementing an extension of scalars from $\mathbb{B}$ to $\mathbb{F}$, and moreover it remains finite of cardinality $n$. One derives the definition of a full functor

$$
\mathbb{P}: \mathcal{P}_{\mathbb{F}} \longrightarrow \mathfrak{F i n}, \quad \mathbb{P}(E)=(E \backslash\{0\}) / \mathbb{F}^{\times},
$$

which associates to a semimodule $E$ over $\mathbb{F}$ the finite quotient space (set) $\mathbb{P}(E)$.

(b) It is important not to confuse the semimodule $\mathbb{F}^{(n)}$ with the induced module $\mathbb{F} \otimes_{\mathbb{B}} \mathbb{B}^{(n, 1)}$ that can be realized as the range $P\left(\mathbb{F}^{n}\right)$ of the projection $P$ as in (11) promoted to an element of $M_{n}(\mathbb{F})$. There exists a unique map $\phi_{n}: \mathbb{F} \times \mathbb{B}^{(n, 1)} \rightarrow \mathbb{F}^{(n)}=(\mathbb{Z}, \theta)^{\vee}$ which vanishes whenever one of the two arguments does so and is defined as follows:

$$
\begin{aligned}
\phi_{n}: \mathbb{F} \times \mathbb{B}^{(n, 1)} \rightarrow \mathbb{F}^{(n)} & =(\mathbb{Z}, \theta)^{\vee}, \\
\phi_{n}\left(u^{k}, j\right) & =\theta^{k}(j)=j+k n, \quad \forall j \in\{0, \ldots, n-1\} .
\end{aligned}
$$

One has $\phi_{n}(x, i \vee j)=\phi_{n}(x, i) \vee \phi_{n}(x, j) \forall i, j \in\{0, \ldots, n-1\}$ and $x \in \mathbb{F}$. Also $\phi_{n}(x \vee y, i)=\phi_{n}(x, i) \vee \phi_{n}(y, i) \forall i \in\{0, \ldots, n-1\}$ and $x, y \in \mathbb{F}$. Moreover, $\phi_{n}$ is $\mathbb{F}$-linear; that is, one has $\phi_{n}(\lambda x, j)=\lambda \phi_{n}(x, j) \forall \lambda \in \mathbb{F}$. Note that there are more relations in $\mathbb{F}^{(n)}$ than those holding in $\mathbb{F} \otimes_{\mathbb{B}} \mathbb{B}^{(n, 1)}$, for example, the relation $\phi(1, i) \vee \phi(u, j)=\phi(u, j) \forall i, j \in\{0, \ldots, n-1\}$. The semimodule $\mathbb{F}^{(n)}$, when viewed as a semimodule over $\mathbb{B}$, has free rank equal to 1 $\left(\underline{\mathrm{rk}}_{\mathbb{B}}\left(\mathbb{F}^{(n)}\right)=1\right)$, while a similar conclusion fails to hold, as soon as $n>1$, for the semimodule $\mathbb{B}^{(n, 1)} \otimes_{\mathbb{B}} \mathbb{F}$.

As a set, $\mathbb{F}^{(n)}$ is the smash product $\mathbb{F} \wedge \mathbb{B}^{(n, 1)}$ and its additive structure is given by the lexicographic order on the nonzero elements. One needs to clarify in which sense this lexicographic smash product plays the role of the tensor product $\otimes$ for $\mathbb{B}$-semimodules of free rank 1 .

Table 1 summarizes the geometric interpretation of the three categories $\Delta \subset \Lambda \subset \tilde{\Lambda}$ in terms of the geometric categories $\mathcal{P}_{\mathbb{B}} \subset \mathcal{P}_{\mathbb{F}}^{1} \subset \mathcal{P}_{\mathbb{F}}\left(\mathbb{F}=\mathbb{Z}_{\max }\right)$.

\subsection{The perfection of $\mathbb{Z}_{\max }$}

Let $\mathbb{F}^{\mathrm{pf}}=\mathbb{Q}_{\max }:=(\mathbb{Q} \cup\{-\infty\}, \max ,+)$ be the subsemifield of $\mathbb{R}_{+}^{\max }$ (written multiplicatively) containing the (sub)semifield $\mathbb{F}=\mathbb{Z}_{\max }$ (generated by the element $u>1$ of $\mathbb{R}_{+}^{\max }$ ) as well as all rational powers $u^{\alpha}, \alpha \in \mathbb{Q}$.

Recall that a semifield $K$ of characteristic one is called perfect when the map $x \mapsto x^{n}$ is surjective $\forall n \in \mathbb{N}^{\times}$. The map $\operatorname{Fr}_{n}: x \mapsto x^{n}$ defines an 
Table 1

\begin{tabular}{|c|c|}
\hline Projective geometry $\mathcal{P}_{\mathbb{B}}$ over $\mathbb{B}$ & Simplicial category $\Delta \cong \mathcal{P}_{\mathbb{B}}$ \\
\hline Projective geometry $\mathcal{P}_{\mathbb{F}}^{1}$ over $\mathbb{F}$ (linear) & Cyclic category $\Lambda \cong \mathcal{P}_{\mathbb{F}}^{1}$ \\
\hline Projective geometry $\mathcal{P}_{\mathbb{F}}$ over $\mathbb{F}$ (semilinear) & Epicyclic category $\tilde{\Lambda} \cong \mathcal{P}_{\mathbb{F}}$ \\
\hline
\end{tabular}

automorphism of $K$, and one obtains an action $\mathrm{Fr}$ of the multiplicative group $\mathbb{Q}_{+}^{\star}$ on $K$ such that $\operatorname{Fr}_{\alpha}=\operatorname{Fr}_{n} \circ \operatorname{Fr}_{m}^{-1}$ for $\alpha=n / m$.

The following statement summarizes the main properties of $\mathbb{F}^{\mathrm{pf}}=\mathbb{Q}_{\max }$.

\section{Proposition 3.18.}

(i) The semifield $\mathbb{F}^{\mathrm{pf}}$ is perfect, contains $\mathbb{F}$, and for any perfect semifield $K \supset \mathbb{F}$ one derives a canonical homomorphism $\mathbb{F}^{\mathrm{pf}} \rightarrow K$ extending the inclusion $\mathbb{F} \subset K$.

(ii) Any finitely generated subsemifield of $\mathbb{F}^{\mathrm{pf}}$ containing $\mathbb{F}$ is the inverse image $\operatorname{Fr}_{n}^{-1}(\mathbb{F})=\operatorname{Fr}_{1 / n}(\mathbb{F})$ of $\mathbb{F}$ for some integer $n \in \mathbb{N}$. For $m, n \in \mathbb{N}$ one has $\operatorname{Fr}_{n}^{-1}(\mathbb{F}) \subset \mathrm{Fr}_{m}^{-1}(\mathbb{F})$ if and only if $n \mid m$.

(iii) The intersection of the semifields $\operatorname{Fr}_{m}(\mathbb{F}) \forall m \in \mathbb{N}$ is the semifield $\mathbb{B}$.

Proof. (i) A semifield $K$ of characteristic one is perfect if and only if its multiplicative group $K^{\times}$is uniquely divisible. This fact implies (i) since $\mathbb{Q} \supset \mathbb{Z}$ is the uniquely divisible closure of $\mathbb{Z}$.

(ii) Let $K \subset \mathbb{F}^{\text {pf }}$ be a finitely generated subsemifield of $\mathbb{F}^{\text {pf }}$ containing $\mathbb{F}$. Since sums $s=\sum b_{j}$ of elements of $\mathbb{F}^{\text {pf }}$ give one of the $b_{j}$, the multiplicative subgroup $K^{\times} \subset K$ is a finitely generated subgroup of $\left(\mathbb{F}^{\mathrm{pf}}\right)^{\times}=\mathbb{Q}$, and thus is of the form $\frac{1}{n} \mathbb{Z} \subset \mathbb{Q}$. This implies (ii).

The proof of (iii) is immediate.

REMARK 3.19. In a semifield $K$ of characteristic one, $x=1$ is the only solution of the equation $x^{n}=1$ since the endomorphism $\operatorname{Fr}_{n}$ is injective. It follows that for any proper extension $K \supsetneq \mathbb{F}^{\text {pf }}$, the group $K^{\times} / \mathbb{F}^{\text {pf }}$ is infinite and torsion-free. Indeed $K^{\times}$is torsion-free and the group $\left(\mathbb{F}^{\mathrm{pf}}\right)^{\times}=\mathbb{Q}$ is divisible, thus $K^{\times} / \mathbb{F}^{p^{\times}}$is also torsion-free. 


\section{$\S 4$. Duality}

One key property of the cyclic category $\Lambda$ is that it is anti-isomorphic to itself, that is, one has a contravariant functor $\Lambda \longrightarrow \Lambda, f \mapsto f^{t}$, that determines an isomorphism $\Lambda \cong \Lambda^{\mathrm{op}}$. In this section we show that this duality corresponds to transposition of linear maps in the framework of projective geometry in characteristic one as developed in Section 3.

\subsection{Self-duality of $\Lambda$}

\section{Proposition 4.1.}

(i) Let $a, b>0$ be integers, and let $f: \mathbb{Z} \rightarrow \mathbb{Z}$ be a nondecreasing map such that $f(x+a)=f(x)+b$ for all $x \in \mathbb{Z}$. Then there exists a unique map $f^{t}: \mathbb{Z} \rightarrow \mathbb{Z}$ such that

$$
f(x) \geq y \Longleftrightarrow x \geq f^{t}(y), \quad \forall x, y \in \mathbb{Z} .
$$

Moreover, $f^{t}$ is nondecreasing and fulfills $f^{t}(x+b)=f^{t}(x)+a$ for all $x \in \mathbb{Z}$.

(ii) Let $h \in \operatorname{Hom}_{\Lambda}([n],[m])$. Then the class of $f^{t}$ is independent of the choice of $f \in h$ and defines an element $h^{t} \in \operatorname{Hom}_{\Lambda}([m],[n])$.

(iii) The association $h \mapsto h^{t}$ defines a contravariant functor $\Lambda \longrightarrow \Lambda$.

Proof. (i) The inverse image $f^{-1}(I)$ of an interval $I \subset \mathbb{Z}$ of length $b$ is an interval $J$ of length $a$, since the translates $I+k b$ form a partition of $\mathbb{Z}$ and $f^{-1}(I+k b)=J+k a$. It follows that $f^{-1}([y, \infty))$ is of the form $\left[f^{t}(y), \infty\right)$ for a uniquely defined map $f^{t}: \mathbb{Z} \rightarrow \mathbb{Z}$. For $y \leq y^{\prime}$ one has $f^{-1}\left(\left[y^{\prime}, \infty\right)\right) \subset$ $f^{-1}([y, \infty))$ and thus $f^{t}\left(y^{\prime}\right) \geq f^{t}(y)$, so that $f$ is nondecreasing. Moreover, the equality $f^{-1}(I+k b)=J+k a$ shows that $f^{t}(x+b)=f^{t}(x)+a \forall x \in \mathbb{Z}$.

(ii) Let $f: \mathbb{Z} \rightarrow \mathbb{Z}$ be as in (i). Let $k \in \mathbb{Z}$ and $f^{\prime}$ be given by $f^{\prime}(x)=$ $f(x)-k b \forall x \in \mathbb{Z}$. Then one has for any interval $I \subset \mathbb{Z}$ of length $b, f^{\prime-1}(I)=$ $f^{-1}(I+k b)=J+k a$. This shows that $f^{\prime t}(y)=f^{t}(y)+k a \forall y \in \mathbb{Z}$. Taking $a=n+1, b=m+1$, and using (i), one obtains the required statement.

(iii) This follows from the equalities $(f \circ g)^{-1}[y, \infty)=g^{-1}\left(f^{-1}[y, \infty)\right)=$ $g^{-1}\left[f^{t}(y), \infty\right)=\left[g^{t}\left(f^{t}(y)\right), \infty\right)$ which show that $(f \circ g)^{t}=g^{t} \circ f^{t}$.

Let $\mathcal{C}$ be a small category, and let $\alpha$ be a map which assigns to each object $j$ of $\mathcal{C}$ an element $\alpha(j) \in \operatorname{Aut}_{\mathcal{C}}(j)$. We then denote by $\operatorname{Ad}(\alpha) \in \operatorname{Aut}(\mathcal{C})$ the automorphism which is the identity on objects and is given on morphism by

$$
\operatorname{Ad}(\alpha)(h):=\alpha(j) \circ h \circ \alpha(i)^{-1}, \quad \forall h \in \operatorname{Hom}_{\mathcal{C}}(i, j) .
$$


We call such automorphisms inner, and we endow the group $\operatorname{Aut}(\mathcal{C})$ of automorphisms of $\mathcal{C}$ with the topology of pointwise convergence for the discrete topology.

\section{Proposition 4.2.}

(i) The covariant functor $\Lambda \longrightarrow \Lambda$, which is the square of $h \mapsto h^{t}$, is equivalent to the identity by the natural transformation implemented by the $\operatorname{map}[n] \mapsto \tau \in \operatorname{Aut}_{\Lambda}([n]), \tau(x)=x-1 \forall x \in \mathbb{Z}$.

(ii) Let $\operatorname{Ad}(\tau) \in \operatorname{Aut}(\Lambda)$ be the inner automorphism obtained as in (18) using the map $[n] \mapsto \tau \in \operatorname{Aut}_{\Lambda}([n])$. The action of the group $\mathbb{Z}$ on $\Lambda$ : $j \mapsto \operatorname{Ad}(\tau)^{j}$ extends to a continuous action of the profinite completion $\hat{\mathbb{Z}}, \alpha: \hat{\mathbb{Z}} \rightarrow \operatorname{Aut}(\Lambda)$.

(iii) An automorphism $\gamma \in \operatorname{Aut}(\Lambda)$ belongs to $\alpha(\hat{\mathbb{Z}})$ if and only if it is inner and its unique extension to $\tilde{\Lambda}$ as an inner automorphism fixes the $\psi_{k}$ of (5).

Proof. (i) Let $h \in \operatorname{Hom}_{\Lambda}([n],[m])$, and let $f \in h$. Then $f^{t} \in h^{t}$ so that (17) holds. Using (17) one obtains $f(x)<y \Longleftrightarrow x<f^{t}(y) \forall x, y \in \mathbb{Z}$, or equivalently $f^{t}(y) \geq x+1 \Longleftrightarrow y \geq f(x)+1 \forall x, y \in \mathbb{Z}$. One also has $f^{t}(y) \geq$ $x+1 \Longleftrightarrow y \geq\left(f^{t}\right)^{t}(x+1) \forall x, y \in \mathbb{Z}$, which thus gives the equality $\left(f^{t}\right)^{t}(x+$ 1) $=f(x)+1, \forall x \in \mathbb{Z}$.

(ii) Let $z=\left(z_{a}\right)_{a \in \mathbb{N}} \in \prod_{a \in \mathbb{N}} \mathbb{Z} / a \mathbb{Z}$. Then the map $[n] \mapsto \tau^{z_{n+1}} \in \operatorname{Aut}_{\Lambda}([n])$ implements an inner automorphism $\beta(z) \in \operatorname{Aut}(\Lambda)$, and the $\operatorname{map} \beta: \prod_{a \in \mathbb{N}} \mathbb{Z} /$ $a \mathbb{Z} \rightarrow \operatorname{Aut}(\Lambda)$ is a continuous group homomorphism. Composing $\beta$ with the natural inclusion $\hat{\mathbb{Z}} \subset \prod_{a \in \mathbb{N}} \mathbb{Z} / a \mathbb{Z}$, one obtains the required continuous action $\alpha: \hat{\mathbb{Z}} \rightarrow \operatorname{Aut}(\Lambda)$.

(iii) Let $\gamma \in \operatorname{Aut}(\Lambda)$ be an inner automorphism. Then we claim that there exists a unique $z=\left(z_{a}\right)_{a \in \mathbb{N}} \in \prod_{a \in \mathbb{N}} \mathbb{Z} / a \mathbb{Z}$ such that $\gamma=\beta(z)$. Indeed, since every element of $\operatorname{Aut}_{\Lambda}([n])$ is a power $\tau^{z_{n+1}}$, one gets the existence of $z$. The uniqueness follows since the action of $\gamma$ on $\operatorname{Hom}_{\Lambda}([0],[n])$ uniquely determines $z_{n+1}$ modulo $n+1$. For a pair $n, k \in \mathbb{N}$, one has $\psi_{k} \in \operatorname{Hom}_{\mathfrak{A} \mathfrak{r} \triangleleft \mathbb{N} \times}\left(\Psi_{k}(\hat{n})\right.$, $\hat{n})$ and $\tau^{a} \psi_{k}=\psi_{k} \tau^{b} \Longleftrightarrow b \equiv a$ modulo $n$. Thus the extension of the inner automorphism $\gamma=\beta(z)$ to $\tilde{\Lambda}$ fixes the $\psi_{k}$ if and only if

$$
z_{b} \equiv z_{a} \text { modulo } a, \quad \forall b=k a \text {. }
$$

In turn (19) characterizes the elements of the projective limit $\hat{\mathbb{Z}}=\lim \mathbb{Z} / a \mathbb{Z}$, thus (iii) holds. 


\subsection{Duality and transposition for semimodules}

Next we describe the relation between the contravariant functor $\Lambda \longrightarrow \Lambda$, $f \mapsto f^{t}$ and the transposition of morphisms in linear algebra. Transposition is determined in a precise form by implementing the duality for $\mathbb{B}$ semimodules $E$ with $\underline{\mathrm{rk}} E=1$. Recall that for any such $\mathbb{B}$-semimodule $E$, the relation $x \leq y \Longleftrightarrow x \vee y=y$ is a total order on $E$.

Proposition 4.3.

(i) Let $E$ be a $\mathbb{B}$-semimodule with $\underline{\mathrm{rk}} E=1$. Then for any $y \in E$, the following formula defines a linear form $\ell_{y} \in \operatorname{Hom}_{\mathbb{B}}(E, \mathbb{B})$ :

$$
\ell_{y}(x)=\langle x, y\rangle_{\mathbb{B}}:= \begin{cases}0, & \text { if } x \leq y \\ 1, & \text { if } x>y\end{cases}
$$

(ii) For $z, t \in E$, set $z \wedge t:=\inf (z, t)$. The pairing (20) satisfies the following bilinearity property:

$$
\langle x \vee y, z \wedge t\rangle_{\mathbb{B}}=\langle x, z\rangle_{\mathbb{B}}+\langle y, z\rangle_{\mathbb{B}}+\langle x, t\rangle_{\mathbb{B}}+\langle y, t\rangle_{\mathbb{B}}
$$

where + denotes the idempotent addition in $\mathbb{B}$.

(iii) Let $E$ be a $\mathbb{B}$-semimodule with $\underline{\mathrm{rk}} E=1$, and let $\mathrm{rk} E<\infty$. Let $E^{*}$ be the set $E$ endowed with the binary operation $\wedge$ as in (ii). Then $E^{*}$ is a $\mathbb{B}$-semimodule with $\underline{\mathrm{rk}} E^{*}=1, \mathrm{rk} E^{*}=\mathrm{rk} E$. Moreover, the map $y \mapsto \ell_{y}$ defines a $\mathbb{B}$-linear isomorphism $E^{*} \stackrel{\sim}{\rightarrow} \operatorname{Hom}_{\mathbb{B}}(E, \mathbb{B})$.

Proof. (i) One has $\ell_{y}(0)=0$ since $0 \leq y, \forall y \in E$. Moreover, for any two elements $x, x^{\prime} \in E$ the following equality holds: $\left\langle x \vee x^{\prime}, y\right\rangle_{\mathbb{B}}=\langle x, y\rangle_{\mathbb{B}}+$ $\left\langle x^{\prime}, y\right\rangle_{\mathbb{B}}$, since one of these elements is greater than $y$ if and only if the largest of the two is greater than $y$.

(ii) For $y, z \in E$, with $y \wedge z:=\inf (y, z)$ one has $\langle x, y \wedge z\rangle_{\mathbb{B}}=\langle x, y\rangle_{\mathbb{B}}+$ $\langle x, z\rangle_{\mathbb{B}}, \forall x \in E$. Indeed, $\inf (y, z)<x$ if and only if $y<x$ or $z<x$.

(iii) We can view $E$ as a finite totally ordered set. Then $E^{*}$ is the same set but endowed with the opposite ordering so that the largest element of $E$ is the smallest in $E^{*}$, that is, the 0-element for $E^{*}$. It follows that $\underline{\mathrm{rk}} E^{*}=1$, $\operatorname{rk} E^{*}=\operatorname{rk} E$. The map $E^{*} \rightarrow \operatorname{Hom}_{\mathbb{B}}(E, \mathbb{B}), y \mapsto \ell_{y}$ is $\mathbb{B}$-linear by $(21)$. It is injective since $\ell_{y}^{-1}(\{0\})=[0, y]$. We show that it is also surjective. Let $L \in$ $\operatorname{Hom}_{\mathbb{B}}(E, \mathbb{B})$; then $L(0)=0$ and $L$ is a nondecreasing, so $L^{-1}(\{0\})=[0, y]$ for some $y \in E$, and thus $L=\ell_{y}$. 
Proposition 4.3 shows that the duality of $\mathbb{B}$-semimodules $E$ with $\underline{\mathrm{rk}} E=1$ and $\mathrm{rk} E<\infty$ behaves similarly to the duality holding for finite-dimensional vector spaces over fields, and it produces in particular the transposition of linear maps defined as follows. Let $E^{*}=\operatorname{Hom}_{\mathbb{B}}(E, \mathbb{B})$ and $F^{*}=\operatorname{Hom}_{\mathbb{B}}(F, \mathbb{B})$ :

$$
\begin{aligned}
\operatorname{Hom}_{\mathbb{B}}(E, F) & \ni f \mapsto f^{*} \in \operatorname{Hom}_{\mathbb{B}}\left(F^{*}, E^{*}\right), \\
f^{*}(L) & =L \circ f, \quad \forall L \in \operatorname{Hom}_{\mathbb{B}}(F, \mathbb{B}) .
\end{aligned}
$$

As a corollary of Proposition 3.7, one derives the following.

Proposition 4.4. The simplicial category $\Delta$ is canonically isomorphic to the full subcategory of the category of $\mathbb{B}$-semimodules whose objects are the $\mathbb{B}^{(n, 1)}$ for $n \geq 1$, and the morphisms are the linear maps $f$ such that $f^{-1}(\{0\})=\{0\}$.

Although one has a canonical isomorphism $\left(\mathbb{B}^{(n, 1)}\right)^{*} \cong \mathbb{B}^{(n, 1)}$, the condition $f^{-1}(\{0\})=\{0\}$ is not preserved by transposition. In the following discussion we will show that the transposed of the above condition is understood within the category $\mathcal{J}$ of intervals (i.e., totally ordered sets) $I$ with a smallest element $b_{I} \in I\left(b_{I} \leq a, \forall a \in I\right)$ and a largest element $t_{I} \in I$ (see [18, VIII.8]).

The morphisms of the category $\mathcal{J}$ are

$$
\operatorname{Hom}_{\geq}(I, J)=\left\{f: I \rightarrow J \mid x \leq y \Longrightarrow f(x) \leq f(y), f\left(b_{I}\right)=b_{J}, f\left(t_{I}\right)=t_{J}\right\},
$$

namely, nondecreasing maps preserving the two endpoints. Notice that given a $\mathbb{B}$-semimodule $E$ with $\underline{\operatorname{rk}}(E)=1$ and $\operatorname{rk}(E)<\infty$, the underlying ordered set $E_{\leq}$is an interval.

Proposition 4.5. Let $f \in \operatorname{Hom}_{\mathbb{B}}(E, F)$, with $E, F, \mathbb{B}$-semimodules of finite rank and free rank 1 . Then

(i) $f^{-1}(\{0\})=\{0\}$ if and only if $f^{*} \in \operatorname{Hom}_{\leq}\left(F_{\leq}^{*}, E_{\leq}^{*}\right)$;

(ii) $f \in \operatorname{Hom}_{\leq}\left(E_{\leq}, F_{\leq}\right)$if and only if $f^{*} \in \operatorname{Hom}_{\mathbb{B}}\left(F^{*}, E^{*}\right)$ fulfills $\left(f^{*}\right)^{-1}(\{0\})=\{0\}$

(iii) the transposition of maps $f \mapsto f^{*}$ determines an isomorphism of $\Delta^{\mathrm{op}}$ with the full subcategory of $\mathcal{J}$ defined by the intervals of the form $n^{*}:=$ $\{0,1, \ldots, n+1\}$, for $n \geq 0$.

Proof. (i) Let $E$ be a $\mathbb{B}$-semimodule with $\underline{\mathrm{rk}} E=1$ and $\operatorname{rk} E<\infty$. Then $\operatorname{Hom}_{\mathbb{B}}(E, \mathbb{B})$ is an interval whose largest element is the linear form $\tau_{E}$ : 
$\tau_{E}(x)=1 \forall x \in E, x \neq 0$. For $f \in \operatorname{Hom}_{\mathbb{B}}(E, F)$ as in (i), one has $f^{-1}(\{0\})=$ $\{0\}$ if and only if $\tau_{F} \circ f=\tau_{E}$. The smallest element of $\operatorname{Hom}_{\mathbb{B}}(F, \mathbb{B})$ is the linear form 0 which is automatically preserved by composition with any $f \in \operatorname{Hom}_{\mathbb{B}}(E, F)$.

(ii) One has $f \in \operatorname{Hom}_{\leq}\left(E_{\leq}, F_{\leq}\right)$if and only if $f\left(t_{E}\right)=t_{F}$, where $t_{E}$ (resp., $\left.t_{F}\right)$ is the largest element of $E$. This holds if and only if $t_{E} \notin f^{-1}([0, y])$ $\forall y<t_{F}$, that is, if and only if $\left(f^{*}\right)^{-1}(\{0\})=\{0\}$.

(iii) For $n \geq 0$, the dual semimodule $\left(\mathbb{B}^{(n+1,1)}\right)^{*}$ is an interval of cardinality $n+2$, and hence coincides with $n^{*}$. Transposition determines a contravariant functor $\mathcal{P}_{\mathbb{B}} \longrightarrow \mathcal{J}$.

In terms of the isomorphism $E^{*} \rightarrow \operatorname{Hom}_{\mathbb{B}}(E, \mathbb{B}), y \mapsto \ell_{y}$ of Proposition 4.3, the transposed $f^{*}$ of $f \in \operatorname{Hom}_{\mathbb{B}}(E, F)$ replaces $\ell_{y}$ by $\ell_{y} \circ f$, for $y \in F$, and hence is defined by the equation

$$
\langle f(x), y\rangle_{\mathbb{B}}=\left\langle x, f^{*}(y)\right\rangle_{\mathbb{B}}, \quad \forall x \in E, y \in F^{*} .
$$

Using the above notation, we obtain the following description for the basic equation (17):

$$
\langle y, f(x)\rangle_{\mathbb{B}}=\left\langle f^{t}(y), x\right\rangle_{\mathbb{B}}, \quad \forall x, y \in \mathbb{Z} .
$$

This shows that, once interpreted in the framework of characteristic one, the contravariant functor $\Lambda \longrightarrow \Lambda, f \mapsto f^{t}$, is simply inverse transposition.

REMARK 4.6. At first, it might seem puzzling that the transposition $f \mapsto f^{*}$ fulfills $\left(f^{*}\right)^{*}=f$ while the map $f \mapsto f^{t}$ of Proposition 4.5 is not involutive. The reason for this behavior is that for a finite totally ordered set $E$ viewed as a $\mathbb{B}$-semimodule, the dual $E^{*}$ is the same set but endowed with the opposite order: $x \leq^{*} y \Longleftrightarrow y \leq x$. By applying (22) one derives $f^{*}(z) \leq^{*}$ $t \Longleftrightarrow z \leq^{*}\left(f^{*}\right)^{*}(t), \forall z \in F^{*}, t \in E^{*}$ which is equivalent to $t \leq f^{*}(z) \Longleftrightarrow$ $\left(f^{*}\right)^{*}(t) \leq z$. This shows, using $(22)$, that $\left(f^{*}\right)^{*}=f$. When considering the map $f \mapsto f^{t}$ of Proposition 4.5 , one applies the same formula twice, while taking into account the opposite order would provide the inverse of the map $f \mapsto f^{t}$. Since the negation of $x \leq y$ is $x>y$, that is, $y+1 \leq x$, for the ordered set $\mathbb{Z}$, the translation of 1 pops up and conjugates the map $f \mapsto f^{t}$ with its inverse.

Next, we develop the above duality directly at the level of semimodules over $\mathbb{F}=\mathbb{Z}_{\text {max }}$. Let $(X, \theta)$ be an archimedean set, and let $E=(X, \theta)^{\vee}$ be 
the associated semimodule over $\mathbb{F}$ as in (12) and (13). The archimedean property ensures that the following pairing is well defined with values in $\mathbb{F}$ :

$$
\langle x, y\rangle_{\mathbb{F}}:=\inf \{v \in \mathbb{F} \mid x \leq v y\}, \quad \forall x, y \in E, y \neq 0 .
$$

\section{Proposition 4.7.}

(i) Let $(X, \theta)$ be an archimedean set, and let $E=(X, \theta)^{\vee}$ be the associated semimodule over $\mathbb{F}$ (see Proposition 3.9). Let $E^{*}=\left(X^{\prime}, \theta^{-1}\right)^{\vee}$, where $X^{\prime}$ is the set $X$ endowed with the opposite order. Then (24) defines a bilinear pairing $E \times E^{*} \rightarrow \mathbb{F}$.

(ii) Let $X=\mathbb{Z}, \theta(x)=x+n$, and let $E, E^{*}$ be the associated semimodules over $\mathbb{F}$ as in (i). Then (24) determines an isomorphism

$$
\ell: E^{*} \stackrel{\sim}{\rightarrow} \operatorname{Hom}_{\mathbb{F}}(E, \mathbb{F}), \quad \ell_{y}(x):=\langle x, y\rangle_{\mathbb{F}}, \forall x \in E, y \in E^{*}
$$

(iii) The contravariant functor $f \mapsto f^{t}$ from $\Lambda$ to $\Lambda$ is the inverse of transposition $f \mapsto f^{*}$ :

$$
\begin{gathered}
\operatorname{Hom}_{\mathbb{F}}(E, F) \ni f \mapsto f^{*} \in \operatorname{Hom}_{\mathbb{F}}\left(F^{*}, E^{*}\right), \\
\langle f(x), y\rangle_{\mathbb{F}}=\left\langle x, f^{*}(y)\right\rangle_{\mathbb{F}}, \quad \forall x \in E, y \in F^{*} .
\end{gathered}
$$

Proof. (i) For $y \in X$, the archimedean property ensures that the set $\{v \in$ $\mathbb{F} \mid x \leq v y\}$ is nonempty $\forall x \in E$. Thus (24) is well defined and gives $0 \in \mathbb{F}$ only for $x=0 \in E$. For $0 \neq \lambda \in \mathbb{F}$, one has $\{v \in \mathbb{F} \mid \lambda x \leq v y\}=\lambda\{v \in \mathbb{F} \mid$ $x \leq v y\}$, which shows that $\langle\lambda x, y\rangle_{\mathbb{F}}=\lambda\langle x, y\rangle_{\mathbb{F}}$. For $x \leq x^{\prime}$, one has $\{v \in \mathbb{F} \mid$ $\left.x^{\prime} \leq v y\right\} \subset\{v \in \mathbb{F} \mid x \leq v y\}$, thus $\langle x, y\rangle_{\mathbb{F}} \leq\left\langle x^{\prime}, y\right\rangle_{\mathbb{F}}$. This shows that the map $x \mapsto\langle x, y\rangle_{\mathbb{F}} \in \mathbb{F}$ is $\mathbb{F}$-linear. For $x, y \in E$ and $y \neq 0$, one has for $0 \neq \lambda \in \mathbb{F}$ : $\langle x, \lambda y\rangle_{\mathbb{F}}=\lambda^{-1}\langle x, y\rangle_{\mathbb{F}}$, which corresponds to the $\mathbb{F}$-linearity in $E^{*}$. Also one has $\left\langle x, y^{\prime}\right\rangle_{\mathbb{F}} \leq\langle x, y\rangle_{\mathbb{F}}$ for $y \leq y^{\prime}$ for $y, y^{\prime} \in E$. This corresponds to the linearity in $E^{*}$. Thus the pairing (24) is bilinear.

(ii) It follows from (i) that the map $\ell: E^{*} \rightarrow \operatorname{Hom}_{\mathbb{F}}(E, \mathbb{F})$ is well defined and linear. Let $L \in \operatorname{Hom}_{\mathbb{F}}(E, \mathbb{F})$. We show that there exists a unique $y \in E^{*}$ with $\ell_{y}=L$. This holds for $L=0$, thus we can assume that $L\left(x_{0}\right) \neq 0$ for some $x_{0} \in E$. The archimedean property implies that the kernel of $L$, that is, $\{x \in E \mid L(x)=0\}$, is reduced to $\{0\}$ since for any $x \in E$ there exists $\lambda \in \mathbb{F}$ with $x \geq \lambda x_{0}$ so that $L(x) \geq \lambda L\left(x_{0}\right) \neq 0$. Replacing $L$ by a multiple $\mu L$ for some $\mu \neq 0$, we can thus assume that $L$ corresponds, at the level of archimedean sets, to a nondecreasing map $f: \mathbb{Z} \rightarrow \mathbb{Z}$ with $f(x+n)=$ $f(x)+1 \forall x \in \mathbb{Z}$ and that $f(0)=0$. One then has $f(n)=1$, and $f$ is uniquely 
determined by the element $y \in[0, n-1]$ such that $f(x)=0 \Longleftrightarrow x \leq y$. We show that $L=\ell_{y}$; that is, that for any $x \in \mathbb{Z}, f(x)=g(x)$ where $g(x)$ is the smallest integer $k \in \mathbb{Z}$ such that $x \leq y+k n$. Since $g(x+n)=g(x)+1, \forall x \in \mathbb{Z}$, it is enough to prove that $f(x)=g(x)$ for $x \in[0, n-1]$. For $x \in[0, y]$, one gets $g(x)=0$, since $y-n<x \leq y$. For $x \in[0, n-1], x>y$, one has $g(x)=1$, since $y<x \leq y+n$. This shows that $L=\ell_{y}$ for a unique $y \in \mathbb{Z}$ and thus that the $\operatorname{map} \ell: E^{*} \rightarrow \operatorname{Hom}_{\mathbb{F}}(E, \mathbb{F})$ is bijective.

(iii) The equality

$$
\Re_{\mathbb{B}}(x)=\left\{\begin{array}{ll}
0, & \text { if } x \leq 1, \\
1, & \text { if } x>1,
\end{array} \quad \forall x \in \mathbb{F}\right.
$$

defines a linear form $\Re_{\mathbb{B}}: \mathbb{F} \rightarrow \mathbb{B}$. Moreover, for $(X, \theta)$ an archimedean set and $E=(X, \theta)^{\vee}$ the associated semimodule over $\mathbb{F}$, one has

$$
\langle x, y\rangle_{\mathbb{B}}=\Re_{\mathbb{B}}\left(\langle x, y\rangle_{\mathbb{F}}\right), \quad \forall x, y \in E, y \neq 0 .
$$

Indeed, $x \leq y$ if and only if $1 \in\{v \in \mathbb{F} \mid x \leq v y\}$. By construction, the transposition $\operatorname{Hom}_{\mathbb{F}}(E, F) \ni f \mapsto f^{*} \in \operatorname{Hom}_{\mathbb{F}}\left(F^{*}, E^{*}\right)$ fulfills (25). Applying $\Re_{\mathbb{B}}$ to both sides of (25), one obtains

$$
\langle f(x), y\rangle_{\mathbb{B}}=\left\langle x, f^{*}(y)\right\rangle_{\mathbb{B}}, \quad \forall x \in E, y \in F^{*}
$$

and this shows, using (23), that $\left(f^{*}\right)^{t}=f$.

REMARK 4.8. Let $(X, \theta)$ be an archimedean set, and let $E=(X, \theta)^{\vee}$ be the associated semimodule over $\mathbb{F}$. Let $E^{*}=\left(X^{\prime}, \theta^{-1}\right)^{\vee}$, where $X^{\prime}$ is the set $X$ endowed with the opposite order. It is not true in general that the archimedean sets $(X, \theta)$ and $\left(X^{\prime}, \theta^{-1}\right)$ are isomorphic. But this holds for archimedean sets of the form $(\mathbb{Z}, \theta)$ as in Proposition 4.7(ii). The choice of an isomorphism $\sigma:(X, \theta) \rightarrow\left(X^{\prime}, \theta^{-1}\right)$ yields the notion of quadratic form on the associated semimodule $E=(X, \theta)^{\vee}$ over $\mathbb{F}$. One can then obtain, using a choice of $\sigma$ for $(\mathbb{Z}, \theta)$, the analogue in the above setup of characteristic one, of the geometries of type $\mathrm{B}_{n}$ and $\mathrm{D}_{n}$. We will not pursue this line here but only point out that it fits perfectly with the theory developed in [19, Section 13] through the involution that $\sigma$ determines on the associated projective space $\mathbb{P}(E)$. 


\subsection{Lift of permutations and cyclic descent number}

Following a traditional point of view, the symmetric group $S_{n}$ is interpreted as the limit for $q \rightarrow 1$ of the general linear group $\mathrm{GL}_{n}\left(\mathbb{F}_{q}\right)$ over a finite field $\mathbb{F}_{q}$. Note that in the limit, the cardinality of the projective space $\mathbb{P}^{n-1}\left(\mathbb{F}_{q}\right)$ becomes $n$. In Proposition 2.10 we proved that $\mathfrak{F}_{1}$ extends to a functor $\mathfrak{F}: \mathfrak{A} \mathfrak{r} \triangleleft \mathbb{N}^{x} \longrightarrow$ Sets. When interpreted in terms of geometry over the semifield $\mathbb{F}=\mathbb{Z}_{\max }$, this functor associates to a semimodule $E$ over $\mathbb{F}$ the quotient set $(E \backslash\{0\}) / \mathbb{F}^{\times}$. Now we restrict this functor to the epicyclic category $\tilde{\Lambda}$, that is, to the semimodules $\mathbb{F}^{(n)}$ obtained from the one-dimensional free vector space $\mathbb{F}$ by restriction of scalars as explained in Theorem 3.15. Note that since the semigroup of injective endomorphisms of $\mathbb{F}$ is canonically isomorphic to $\mathbb{N}^{\times}$, the functor Mod restricts to a functor $\operatorname{Mod}: \mathcal{P}_{\mathbb{F}} \longrightarrow \mathbb{N}^{\times}$, where $\mathbb{N}^{\times}$is viewed as a category with a single object.

Definition 4.9 (see [17, Section 4.6.1]). Let $\sigma \in \operatorname{Hom}_{\mathfrak{F i n}}([n],[m])$ be a map of sets. Then the cyclic descent number of $\sigma$ is defined to be

$$
\operatorname{cdesc}(\sigma)=\#\{j \in\{0,1, \ldots, n\} \mid \sigma(j+1)<\sigma(j)\},
$$

where we identify $n+1 \sim 0$.

The following result provides a geometric interpretation of the cyclic descent number of an arbitrary permutation as the measure of its semilinearity.

Proposition 4.10.

(i) The functor $\mathbb{P}: \mathcal{P}_{\mathbb{F}} \longrightarrow \mathfrak{F i n}$ is full.

(ii) Let $\sigma \in \operatorname{Hom}_{\mathfrak{F i n}}([n],[m])$. Then $\operatorname{cdesc}(\sigma)$ is the smallest integer $k$ such that there exists $f \in \operatorname{Hom}_{\mathcal{P}_{\mathbb{F}}}\left(\mathbb{F}^{(n)}, \mathbb{F}^{(m)}\right), \operatorname{Mod}(f)=k$ with $\mathbb{P}(f)=\sigma$.

(iii) Let $\sigma \in \operatorname{Hom}_{\mathfrak{F} \mathfrak{i n}}([n],[m])$ with $\operatorname{cdesc}(\sigma)=k$. Then there exists a unique $f \in \operatorname{Hom}_{\mathcal{P}_{\mathbb{F}}}\left(\mathbb{F}^{(n)}, \mathbb{F}^{(m)}\right), \operatorname{Mod}(f)=k$ such that $\mathbb{P}(f)=\sigma$.

Proof. It is enough to prove (ii) and (iii). Let $U_{k}$ be the set of $f \in$ $\operatorname{Hom}_{\mathfrak{A} \mathfrak{r} \triangleleft \mathbb{N} \times}([n],[m]), \rho(f)=k$ (see Proposition 2.7(ii)) such that $\mathfrak{F}(f)=\sigma$. Using Theorem 3.15, it is enough to show that $U_{k}=\varnothing$ for $k<\operatorname{cdesc}(\sigma)$ and that if $k=\operatorname{cdesc}(\sigma)$, then $U_{k}$ contains a single element. By construction (see Definition 2.6), the elements of $U_{k}$ are equivalence classes of nondecreasing maps, modulo the addition of a constant multiple of $m+1, f: \mathbb{Z} \rightarrow \mathbb{Z}$ such that

(a) $f(x+(n+1))=f(x)+k(m+1) \forall x \in \mathbb{Z}$,

(b) $f(x) \in \sigma(x)+(m+1) \mathbb{Z} \forall x \in\{0,1, \ldots, n, n+1\}, \sigma(n+1):=\sigma(0)$. 
In each equivalence class there is a unique representative $f$ such that $f(0)=\sigma(0)$ : in the following we assume this normalization condition. We let

$$
\begin{aligned}
c(x)= & \#\{j \in\{0,1, \ldots, x-1\} \mid \\
& \sigma(j+1)<\sigma(j)\}, \quad \forall x \in\{0,1, \ldots, n, n+1\},
\end{aligned}
$$

where by convention we set $\sigma(n+1):=\sigma(0)$ so that $c(n+1)=\operatorname{cdesc}(\sigma)$. For $x \in\{0,1, \ldots, n, n+1\}$, let $b(x) \in \mathbb{Z}$ such that $f(x)=\sigma(x)+(m+1) b(x)$. One has $b(0)=0$, and since $f(1) \geq f(0)$, we get $b(1) \geq c(1)$. More generally, for $j \in\{0,1, \ldots, n\}$, one obtains

$$
f(j+1) \geq f(j) \Longrightarrow b(j+1)-b(j) \geq c(j+1)-c(j) .
$$

Indeed, one has $(m+1)(b(j+1)-b(j)) \geq(\sigma(j)-\sigma(j+1))$ and this implies

$$
\mathbb{Z} \ni b(j+1)-b(j) \geq \frac{\sigma(j)-\sigma(j+1)}{m+1}>-1 .
$$

If $\sigma(j+1)<\sigma(j)$, then $b(j+1)-b(j) \geq 1=c(j+1)-c(j)$.

The inequalities $b(j+1)-b(j) \geq c(j+1)-c(j)$ for $j \in\{0,1, \ldots, n\}$ together with $b(0)=c(0)=0$ show that $b(n+1) \geq c(n+1)$. By (a) one has $b(n+$ $1)=k$, while $c(n+1)=\operatorname{cdesc}(\sigma)$. Thus $U_{k}=\varnothing$ for $k<\operatorname{cdesc}(\sigma)$. Moreover, for $k=\operatorname{cdesc}(\sigma)$, all the inequalities $b(j+1)-b(j) \geq c(j+1)-c(j)$ for $j \in\{0,1, \ldots, n\}$ become equalities and we obtain

$$
f(x)=\sigma(x)+(m+1) c(x), \quad \forall x \in\{0,1, \ldots, n\},
$$

which provides the required uniqueness, thus $U_{k}$ contains at most one element. Moreover, one easily checks that the function $f$ defined by (27) on $\{0,1, \ldots, n\}$ and extended by periodicity using (a) is nondecreasing and belongs to $U_{k}$.

\section{$\S 5$. Extension of scalars $-\otimes_{\mathbb{B}} \mathbf{S}$ to hyperfields}

The algebraic constructions discussed in the earlier sections for semifields are in fact the positive part of a general picture that one can elaborate in terms of hyperfields. The development of this translation into the framework of hyperstructures allows one to link with the results of [9], where it was shown that by implementing the theory of hyperstructures one can parallel successfully Fontaine's p-adic arithmetic theory of "perfection" and 
subsequent Witt extension by combining a process of dequantization (to characteristic one) and a consecutive Witt construction (to characteristic zero). It turns out that the semimodules implemented over the semifields $\mathbb{B}$ and $\mathbb{F}$ of the last sections fulfill precisely the property (28) below that allows one to apply the symmetrization process introduced in [16]. This procedure associates to a commutative monoid $M$ such that

$$
\begin{aligned}
& \forall x, y, u, v \in M, x+y=u+v \\
& \Longrightarrow \exists z \in M, \quad\left\{\begin{array}{lr}
x+z=u, & z+v=y, \\
\text { or } x=u+z, & v=z+y
\end{array}\right.
\end{aligned}
$$

a hypergroup $s(M)$, which is the universal solution to the embedding of $M$ into a hypergroup.

It is shown in [16] that the condition (28) is equivalent to the existence of a common refinement of any two decompositions of an element of $M$ as a sum.

Let $E$ be a semimodule over $\mathbb{B}$. Then using the results of [16] one shows that $E$, as a monoid, fulfills (28) if and only if $\underline{\mathrm{rk}} E=1$. Moreover, in [16], it is also proven that the hypergroup $s(E)$, which is the universal solution to the embedding of $E$ into a hypergroup, coincides with the tensor product $E \otimes_{\mathbb{B}} \mathbf{S}$ which we now describe in detail. Let $E$ be a semimodule over $\mathbb{B}$ such that $\underline{\mathrm{rk}} E=1$. We denote by $E \otimes_{\mathbb{B}} \mathbf{S}$ the quotient of $E \times\{ \pm 1\}$ by the equivalence relation that identifies $(0,-1) \sim(0,1)$. We use the notation $\pm x$ for the elements of $E \times\{ \pm 1\}$, and we denote by $x \mapsto|x|$ the projection from $E \otimes_{\mathbb{B}} \mathbf{S}$ to $E$, so that $| \pm x|:=x$. We endow $E \otimes_{\mathbb{B}} \mathbf{S}$ with the multivalued binary operation (here we use the total order $<$ of $E$ )

$$
x \smile y= \begin{cases}x, & \text { if }|x|>|y| \text { or } x=y ; \\ y, & \text { if }|x|<|y| \text { or } x=y ; \\ {[-x, x],} & \text { if } y=-x\end{cases}
$$

where the interval $[-x, x]$ is defined to be the set $\left\{z \in E \otimes_{\mathbb{B}} \mathbf{S}|| z|\leq| x \mid\right\}$.

The following lemma lists several properties inherent to the above-defined tensor product.

Lemma 5.1. Let $E, F$ be semimodules over $\mathbb{B}$ such that $\underline{\mathrm{rk}} E=\underline{\mathrm{rk}} F=1$. 
(i) The tensor product $E \otimes_{\mathbb{B}} \mathbf{S}$ is a canonical hypergroup and a module over $\mathbf{S}$ in the following sense:

$$
\begin{aligned}
& \lambda\left(v \smile v^{\prime}\right)=\lambda v \smile \lambda v^{\prime}, \\
&\left(\lambda+\lambda^{\prime}\right) v \subset \lambda v \smile \lambda^{\prime} v, \quad \forall \lambda, \lambda^{\prime} \in \mathbf{S}, \forall v, v^{\prime} \in E \otimes_{\mathbb{B}} \mathbf{S} .
\end{aligned}
$$

(ii) Let $f: E \rightarrow F$ be a morphism of $\mathbb{B}$-semimodules. Then the following formula defines a morphism of $\mathbf{S}$-modules:

$f \otimes_{\mathbb{B}} \operatorname{id}_{\mathbf{S}}: E \otimes_{\mathbb{B}} \mathbf{S} \rightarrow F \otimes_{\mathbb{B}} \mathbf{S}, \quad f \otimes_{\mathbb{B}} \operatorname{id}_{\mathbf{S}}( \pm x):= \pm f(x), \quad \forall x \in E$.

(iii) Let $\epsilon:(E \backslash\{0\}) \rightarrow\{ \pm 1\}$ be a map of sets. Then the following defines an automorphism $\tilde{\epsilon}: E \otimes_{\mathbb{B}} \mathbf{S} \rightarrow E \otimes_{\mathbb{B}} \mathbf{S}$ (as a module over $\mathbf{S}$ ):

$$
\tilde{\epsilon}( \pm x):= \pm \epsilon(x) x, \quad \forall x \in E
$$

(iv) Let $g \in \operatorname{Hom}_{\mathbf{S}}\left(E \otimes_{\mathbb{B}} \mathbf{S}, F \otimes_{\mathbb{B}} \mathbf{S}\right)$ be a morphism of $\mathbf{S}$-modules such that $g^{-1}(\{0\})=\{0\}$. Then there exists a unique pair $(f, \epsilon)$ of a morphism of $\mathbb{B}$-semimodules $f: E \rightarrow F$ and a map of sets $\epsilon:(E \backslash\{0\}) \rightarrow\{ \pm 1\}$ such that $g=\left(f \otimes_{\mathbb{B}} \operatorname{id}_{\mathbf{S}}\right) \circ \tilde{\epsilon}$.

Proof. (i) We recall (see [6]) that the definition of a canonical hypergroup $H$ requires that $H$ have a neutral element $0 \in H$ (i.e., an additive identity) and that the following axioms apply:

(1) $x+y=y+x, \forall x, y \in H$;

(2) $(x+y)+z=x+(y+z), \forall x, y, z \in H$;

(3) $0+x=x=x+0, \forall x \in H$;

(4) $\forall x \in H \exists ! y(=-x) \in H$ s.t. $0 \in x+y$;

(5) $x \in y+z \Longrightarrow z \in x-y$.

Axioms (1), (3), and (4) are easy to verify for $E \otimes_{\mathbb{B}} \mathbf{S}$. To check (2), note that if among $|x|,|y|,|z|$ only one, say, $|x|$, is strictly larger than the others, then both sides of $(2)$ give $x$. For $|y| \leq|x|$, one has $y \smile[-x, x]=[-x, x]$ since $y \smile[-x, x]$ contains $[-y, y]$ and any $z$ such that $|y|<|z| \leq|x|$. It follows that (2) holds when, among $|x|,|y|,|z|$ two are equal and strictly larger than the remaining one. Finally, when $|x|=|y|=|z|$, both sides give $[-x, x]$ except when $x=y=z$, in which case they both give $x$. The condition (5) follows from the first four since both sides are equivalent to $0 \in-x+y+z$. This shows that $E \otimes_{\mathbb{B}} \mathbf{S}$ is a canonical hypergroup. Moreover, one has $-x \smile$ $-y=-(x \smile y) \forall x, y \in E \otimes_{\mathbb{B}} \mathbf{S}$, which gives the first equality of (30). One 
finally checks that the second inclusion holds and is in general strict for $\lambda^{\prime}=-\lambda$.

(ii) Let $g: E \otimes_{\mathbb{B}} \mathbf{S} \rightarrow F \otimes_{\mathbb{B}} \mathbf{S}$ be defined by $g( \pm x):= \pm f(x), \forall x \in E$. By construction, $g(\lambda x)=\lambda g(x) \forall \lambda \in \mathbf{S}$ and $\forall x \in E \otimes_{\mathbb{B}} \mathbf{S}$. It remains to check that $g$ is a morphism of hypergroups, that is, that $g(x \smile y) \subset g(x) \smile$ $g(y) \forall x, y \in E \otimes_{\mathbb{B}} \mathbf{S}$. Since $f$ is nondecreasing, $|g(x)| \leq|g(y)|$ if $|x| \leq|y|$. If $|x|<|y| x \smile y=y, g(x \smile y)=g(y) \in g(x) \smile g(y)$ since $v \in u \smile v$ when $|u| \leq|v|$. The only remaining case to consider is when $y=-x$. One has $g(x \smile-x)=g([-x, x]) \subset[-g(x), g(x)]$.

(iii) By construction, $\tilde{\epsilon}(\lambda x)=\lambda \tilde{\epsilon}(x) \forall \lambda \in \mathbf{S}$ and $\forall x \in E \otimes_{\mathbb{B}} \mathbf{S}$. It remains to show that $\tilde{\epsilon}(x \smile y)=\tilde{\epsilon}(x) \smile \tilde{\epsilon}(y) \forall x, y \in E \otimes_{\mathbb{B}} \mathbf{S}$. In fact, one has $|\tilde{\epsilon}(x)|=|x|$ $\forall x \in E \otimes_{\mathbb{B}} \mathbf{S}$. Thus (29) shows the required equality when $|x| \neq|y|$ or when $x=y$. The only remaining case is $y=-x$, and in that case the equality follows from $\tilde{\epsilon}([-x, x])=[-x, x]$.

(iv) The map $f: E \rightarrow F$ is uniquely determined by $f(x):=|g(x)| \forall x \in E$. Since $g^{-1}(\{0\})=\{0\}$, this determines uniquely the map $\epsilon: E \backslash\{0\} \rightarrow\{ \pm 1\}$ such that $g=\left(f \otimes_{\mathbb{B}} \operatorname{id}_{\mathbf{S}}\right) \circ \tilde{\epsilon}$. It only remains to show that $f$ is nondecreasing, that is, that $x \leq y$ implies $|g(x)| \leq|g(y)|$. Assume that $x<y$ and that $|g(x)|>|g(y)|$. By hypothesis, one has $g(x \smile y) \subset g(x) \smile g(y) \forall x, y \in$ $E \otimes_{\mathbb{B}} \mathbf{S}$ and this contradicts (29), which gives $x \smile y=y$ and $g(x) \smile g(y)=$ $g(x)$.

Applying Lemma 5.1 to the semifields $\mathbb{F} \subset \mathbb{F}^{\text {pf }}\left(\mathbb{F}=\mathbb{Z}_{\max }\right)$, one obtains the corresponding hyperfields $\mathbb{F} \otimes_{\mathbb{B}} \mathbf{S} \subset \mathbb{F}^{\text {pf }} \otimes_{\mathbb{B}} \mathbf{S}$. The hyperfield $\mathbb{F}^{\text {pf }} \otimes_{\mathbb{B}} \mathbf{S}$ is perfect and coincides with the perfection of $\mathbb{F} \otimes_{\mathbb{B}} \mathbf{S}$. Using this functorial construction, one can recast the results of the previous sections in terms of hyperfields.

Finally, note that as a set, the projective space $\mathbb{P}(E)$ remains unchanged after shifting to the framework of hyperfields since the multiplicative group of, for example, $\mathbb{F} \otimes_{\mathbb{B}} \mathbf{S}$, is simply the product of $\mathbb{F}^{\times}$by the group of signs $\{ \pm 1\}=\mathbf{S}^{\times}$. Thus for a semimodule $E$ over $\mathbb{F}$, the following equality of sets holds:

$$
\left(\left(E \otimes_{\mathbb{B}} \mathbf{S}\right) \backslash\{0\}\right) /\left(\mathbb{F} \otimes_{\mathbb{B}} \mathbf{S}\right)^{\times}=\mathbb{P}(E)=(E \backslash\{0\}) / \mathbb{F}^{\times} .
$$

Acknowledgments. Consani's work was partially supported by National Science Foundation grant DMS-1069218, and she would like to thank the Collège de France for additional financial support. 


\section{REFERENCES}

[1] N. Bourbaki, Éléments de mathématiques: Algèbre, chapitre II, Actualités Sci. Indust. 1032, Hermann, Paris, 1947. MR 0023803.

[2] D. Burghelea, Z. Fiedorowicz, and W. Gajda, Power maps and epicyclic spaces, J. Pure Appl. Algebra 96 (1994), 1-14. MR 1297435. DOI 10.1016/0022-4049(94)90081-7.

[3] A. Connes, Cohomologie cyclique et foncteurs $\mathrm{Ext}^{n}$, C. R. Acad. Sci. Paris Sér. I Math. 296 (1983), 953-958. MR 0777584.

[4] A. Connes and C. Consani, Schemes over $\mathbb{F}_{1}$ and zeta functions, Compos. Math. 146 (2010), 1383-1415. MR 2735370. DOI 10.1112/S0010437X09004692.

[5] - "Characteristic 1, entropy and the absolute point" in Noncommutative Geometry, Arithmetic, and Related Topics, Johns Hopkins University Press, Baltimore, 2011, 75-139. MR 2907005.

[6] — The hyperring of adèle classes, J. Number Theory 131 (2011), 159-194. MR 2736850. DOI 10.1016/j.jnt.2010.09.001.

[7] - Cyclic structures and the topos of simplicial sets, J. Pure Appl. Algebra 219 (2015), 1211-1235. MR 3282133. DOI 10.1016/j.jpaa.2014.06.002.

[8] - Cyclic homology, Serre's local factors and the $\lambda$-operations, preprint, arXiv: $1211.4239 \mathrm{v} 1$ [math.AG].

[9] - The universal thickening of the field of real numbers, preprint, arXiv: $1202.4377 \mathrm{v} 2$ [math.NT].

[10] B. I. Dundas, T. G. Goodwillie, and R. McCarthy, The Local Structure of Algebraic K-theory, Algebr. Appl. 18, Springer, London, 2013. MR 3013261.

[11] C.-A. Faure and A. Frölicher, Morphisms of projective geometries and semilinear maps, Geom. Dedicata 53 (1994), 237-262. MR 1311317. DOI 10.1007/BF01263998.

[12] J. S. Golan, Semirings and Their Applications, Kluwer Academic, Dordrecht, 1999. MR 1746739. DOI 10.1007/978-94-015-9333-5.

[13] M. Gondran and M. Minoux, Graphs, Dioids and Semirings: New Models and Algorithms, Oper. Res./Comput. Sci. Interfaces Ser. 41, Springer, New York, 2008. MR 2389137.

[14] T. G. Goodwillie, Cyclic homology, derivations, and the free loop space, Topology 24 (1985), 187-215. MR 0793184. DOI 10.1016/0040-9383(85)90055-2.

[15] - personal communication to F. Waldhausen, August 10, 1987.

[16] S. Henry, Symmetrization of monoïds as hypergroups, preprint, arXiv:1309.1963v1 [math.AG].

[17] J.-L. Loday, Cyclic Homology, Grundlehren Math. Wiss. 301, Springer, Berlin, 1998. MR 1600246. DOI 10.1007/978-3-662-11389-9.

[18] S. Mac Lane and I. Moerdijk, Sheaves in Geometry and Logic: A First Introduction to Topos Theory, corrected reprint, Universitext, Springer, New York, 1994. MR 1300636.

[19] J. Tits, Sur les analogues algébriques des groupes semi-simples complexes, Centre Belge de Recherches Mathématiques Établissements Ceuterick, Louvain; Librairie Gauthier-Villars, Paris, 1957, 261-289. MR 0108765. 
Alain Connes

Collège de France

Paris F-75005

France

alain@connes.org

Caterina Consani

Department of Mathematics

Johns Hopkins University

Baltimore, Maryland 21218

USA

kc@math . jhu . edu 